Article

\title{
In Vitro Gastrointestinal Digestion and Colonic Catabolism of Mango (Mangifera indica L.) Pulp Polyphenols
}

\author{
José Luis Ordoñez-Díaz ${ }^{1}$, Alicia Moreno-Ortega ${ }^{1,2}$ (), Francisco Javier Roldán-Guerra ${ }^{1}$, \\ Victor Ortíz-Somovilla ${ }^{1}$, José Manuel Moreno-Rojas ${ }^{1}$ (D) and Gema Pereira-Caro ${ }^{1, *(D)}$ \\ 1 Department of Food Science and Health, Andalusian Institute of Agricultural and Fisheries Research and \\ Training (IFAPA), Alameda del Obispo, Avda. Menéndez-Pidal, s/n, 14004 Córdoba, Spain; \\ josel.ordonez@juntadeandalucia.es (J.L.O.-D.); aliciamorenoortega@hotmail.com (A.M.-O.); \\ franjaviroldan@gmail.com (F.J.R.-G.); victor.ortiz.somovilla@juntadeandalucia.es (V.O.-S.); \\ josem.moreno.rojas@juntadeandalucia.es (J.M.M.-R.) \\ 2 Department of Food Science and Technology, University of Córdoba, Campus Rabanales, Ed. Darwin-anexo, \\ 14071 Córdoba, Spain \\ * Correspondence: mariag.pereira@juntadeandalucia.es; Tel.: +34-671-532-734
}

Received: 14 November 2020; Accepted: 8 December 2020; Published: 10 December 2020

\begin{abstract}
Mango (Mangifera indica L.), a fruit with sensorial attractiveness and extraordinary nutritional and phytochemical composition, is one of the most consumed tropical varieties in the world. A growing body of evidence suggests that their bioactive composition differentiates them from other fruits, with mango pulp being an especially rich and diverse source of polyphenols. In this study, mango pulp polyphenols were submitted to in vitro gastrointestinal digestion and colonic fermentation, and aliquots were analyzed by HPLC-HRMS. The main phenolic compounds identified in the mango pulp were hydroxybenzoic acid-hexoside, two mono-galloyl-glucoside isomers and vanillic acid. The release of total polyphenols increased after the in vitro digestion, with an overall bioaccessibility of $206.3 \%$. Specifically, the most bioaccessible mango polyphenols were gallic acid, 3-O-methylgallic acid, two hydroxybenzoic acid hexosides, methyl gallate, 3,4-dihydroxybenzoic acid and benzoic acid, which potentially cross the small intestine reaching the colon for fermentation by the resident microbiota. After $48 \mathrm{~h}$ of fecal fermentation, the main resultant mango catabolites were pyrogallol, gallic and 3,4-dihydroxybenzoic acids. This highlighted the extensive transformation of mango pulp polyphenols through the gastrointestinal tract and by the resident gut microbiota, with the resultant formation of mainly simple phenolics, which can be considered as biomarkers of the colonic metabolism of mango.
\end{abstract}

Keywords: mango polyphenols; simulated in vitro digestion; bioaccessibility; fecal fermentation; degradation products; catabolic pathway

\section{Introduction}

Mango (Mangifera indica L.), a fruit with sensorial attractiveness and extraordinary nutritional and phytochemical composition [1], is one of the most consumed tropical varieties in the world [2]. Today, mangoes rank sixth in total production among major fruit crops worldwide contributing over 55 million tons per year to the global fruit market [2], with Europe being one of the main destinations of this global trade ( $40 \%$ of imports). Within Europe, Andalusia is the only area with a significant commercial production of tropical fruits thanks to the presence of a subtropical climate in certain regions. These tropical crops, which include mangoes, avocados and cherimoyas as the main ones, have been cultivated in growing regions such as the coast of Granada and Málaga, allowing this 
high-quality product to be accessible to the European market within a few hours, thereby eliminated the need for ship and plane transportation, which can take weeks or even months and involve, in many cases, the collection of underripe fruits [3].

Mangoes contain several essential nutrients including carbohydrates, organic acids, dietary fiber and vitamins and minerals. Sugars including sucrose, fructose and glucose are the predominant soluble sugars in mango, while citric acid and malic acid are the major organic acids present in this fruit [4]. Mangoes also contain substantial amounts of non-essential components known as phytochemicals. Bearing all that in mind, growing evidence suggests that the phytochemical composition of mangoes differentiates them from other fruits, with mango pulp an especially rich and diverse source of phenolic compounds, including mangiferin (C2- $\beta$-D-glucopyranosyl-1,3,6,7-tetrahydroxyxanthone), flavonoids (quercetin, catechin and epicatechin), phenolic acids (benzoic and gallic), and a wide range of derivatives such as ellagic acid, gallotannins and ellagitannins [5]. The bioactivity of the parent polyphenolics found in mangoes has been widely investigated and found to include antimicrobial [6], anti-inflammatory [7], antidiabetic [8,9] and anti-carcinogenic activities [9-11]. Moreover, the health benefits of mangiferin, a singular polyphenol in the mango, has been widely confirmed and continues to attract considerable attention, especially for its potential to reduce the development of degenerative diseases like heart disease and cancer [12,13]. However, little consideration has been given to the impact that human digestion has on bioactivity once a food is consumed. There is now growing evidences that it is not the parent polyphenols of plant foods, rather their metabolites/colonic catabolites that exert health effects in vivo [14]. Moreover, studies into the health benefits of mango have focused mainly on its bark, leaves, peel, and seed/kernel due to their high content of pharmacologically active compounds. In contrast, very little information is available about the flesh/pulp, the part commonly consumed in fresh/processed juices, purees or dried fruits [15]. The polyphenol content of mango pulp is of particular interest given recent clinical observations that sustained consumption of mango pulp (42 days) reduces the systolic blood pressure of lean subjects and maintained the long-term glucose homeostasis in obese subjects. In addition, mango galloyl-derivatives may contribute to the prevention and treatment of obesity and metabolic disorders, benefits that are still to be confirmed [16]. More recently, it has been shown that the consumption of freeze-dried mango pulp can decrease the glucose levels and increase the high-density lipoprotein cholesterol in healthy adults when it is consumed after a high-fat meal [17].

To date, there are two reports describing the in vitro bioaccessibility of mango polyphenols in 'Ataulfo' by-products from pulp paste and peel [18] and in mangoes with different ripening stages [19]. More recently, another two studies have shown the importance of colonic microbiota in the metabolism of mango polyphenols by evaluating the bioaccessibility and colonic fermentation of high-dietary fiber mango-based fruit bars [20] or mango bagasse formulations [21]. To further elucidate the bioaccessibility and colonic transformation of mango pulp polyphenols, this study aims to investigate the stability and resultant breakdown products of mango pulp polyphenols during simulated gastrointestinal digestion and in vitro fecal fermentation by ultra-high-performance liquid chromatography coupled to high-resolution mass spectrometry (UHPLC-HRMS).

\section{Materials and Methods}

\subsection{Chemicals}

Magnesium chloride (hexahydrate), calcium chloride as well as sodium chloride were purchased from Fisher Scientific (Madrid, Spain); ammonium carbonate and sodium bicarbonate were acquired by Sigma-Aldrich (Madrid, Spain); and sodium hydrogen carbonate, potassium dihydrogen phosphate, potassium hydrogen phosphate and magnesium sulfate monohydrate were supplier from VWR International Eurolab (Barcelona, Spain). Yeast extracts, peptone, tween 80, hemin, vitamin K, L-cysteine hydrochloride monohydrate, resazurin redox indicator and calcium chloride were acquired from Sigma-Aldrich. The enzymes used in this study were $\alpha$-amylase (300-1500 U/mg protein) from human 
saliva, pepsin (3.2-4.5 U/mg protein), porcine pancreatin (4xUPS) and bile salts, all from Sigma-Aldrich. $\mathrm{HCl}$ was obtained from Merck (Darmstadt, Germany) and $\mathrm{NaOH}$ from Fisher Scientific (Madrid, Spain). Reference standard compounds including benzene-1,2-diol (pyrogallol), 3,4,5-triydroxybenzoic acid (gallic acid), 3,4-dihydroxybenzoic acid, benzoic acid, methylgallate, 4-hydroxy-3-methoxybenzaldehyde (vanillic acid), quercetin, isorhamnetin, $4^{\prime}$-hydroxy-3'-methoxycinnamic acid (ferulic acid), sinapic acid, $3^{\prime}, 4^{\prime}$-dihydroxycinnamic acid (caffeic acid) and mangiferin were purchased from Sigma-Aldrich. 3-O-Methylgallic acid, 4-O-methylgallic acid, epigallocatechin, epigallocatechin gallate and epicatechin gallate were obtained from Extrasyntheses (France). The acetonitrile and methanol were of LC-MS grade. Note, the nomenclature of the phenolic catabolites used in this paper is based on the recommendation made by Kay et al. [22].

\subsection{Materials and Sample Preparation}

Fresh mangoes (Mangifera indica L. cv. Osteen) were kindly supplied by TROP SAT (Málaga, Spain) which certified the mango species. They were washed and the peel manually removed. Then, they were cut into small pieces and milled using a homogenizer (SAMMIC, Madrid, Spain). Afterwards, the mango puree was lyophilized in a freeze dryer ECO EVO (Tred Technology S.R.L., Ripalimosani, Italy) and stored at $-80^{\circ} \mathrm{C}$.

\subsection{In Vitro Gastrointestinal Digestion}

The in vitro gastrointestinal digestion procedure was performed according to Moreno-Ortega et al. [23]. The whole process consisted in three steps: the oral, gastric and intestinal phases. Briefly, $2 \mathrm{~g}$ of lyophilized sample was firstly submitted to the the oral phase which consisted in $14 \mathrm{~mL}$ of a simulated salivary fluid (SSF), $325 \mu \mathrm{g}$ of $\alpha$-amylase, $0.1 \mathrm{~mL}$ of $0.3 \mathrm{M} \mathrm{CaCl}_{2}$ and distilled water up to $20 \mathrm{~mL}$. The oral mixture was incubated at $37^{\circ} \mathrm{C}$ in a shaking bath (Unitronic Reciprocating Shaking Bath, model 6,032,011, J.P. Selecta, Barcelona, Spain) during $30 \mathrm{~min}$. Then, the mixture was adjusted to $\mathrm{pH} 3$ and submitted to a gastric phase which consisted in $15 \mathrm{~mL}$ of simulated gastric fluids (SGF), $1.19 \mathrm{~mL}$ of $0.1 \mathrm{~g} / \mathrm{mL}$ pepsin solution in $0.1 \mathrm{M}$ HCL, $0.01 \mathrm{~mL}$ of $0.3 \mathrm{M} \mathrm{CaCl}_{2}$ and distilled water up to a final volume of $40 \mathrm{~mL}$. The gastric mixture was incubated at $37^{\circ} \mathrm{C}$ for $120 \mathrm{~min}$. Finally, to carried out the intestinal phase, $22 \mathrm{~mL}$ of simulated intestinal fluids (SIF) together with $80 \mathrm{mg}$ of pancreatin, $125 \mathrm{mg}$ of bile salts, $0.08 \mathrm{~mL}$ of $0.3 \mathrm{M} \mathrm{CaCl}_{2}$ and $9.92 \mathrm{~mL}$ of distilled water were added. Immediately after, the $\mathrm{pH}$ was adjusted to 7 and the whole mixture was incubated at $37^{\circ} \mathrm{C}$ during $120 \mathrm{~min}$ in a shaking bath. The details of the simulated fluids were described in Moreno-Ortega et al. [23].

Samples were taken before (BOD) and after oral digestion (AOD) and after gastric (AGD) and intestinal digestion (AID) using different experiments. These samples were lyophilized and stored at $-80^{\circ} \mathrm{C}$.

\subsection{In Vitro Colonic Fermentation}

The freeze-dried digested mango puree was subjected to in vitro fermentation to simulate the conditions present in the colon following the method described by De Santiago et al. [24] and adapted to our laboratory. Details of the composition of the growth medium are described in De Santiago et al. [24]. The human faecal samples were obtained from three healthy non-smoking volunteers (three males aged between 22 and 34 and with body mass indexes (BMIs) between 20.5 and 25.4). These volunteers had not consumed antibiotics at least 6 months before the study. Forthy eight hours before the faecal sample collection, the volunteers followed a low polyphenol. The samples were collected by the donors in plastic tubes containing an AnaeroGen sachet (Oxoid Ltd., Cambridge, UK) to maintain anaerobic conditions during the transport and were processed within $30 \mathrm{~min}$ of passage. The faeces were homogeneized in pre-reduced phosphate buffered saline (PBS). The temperature of the incubation was set to $37^{\circ} \mathrm{C}$ using a Unitronic OR circulating water-bath (JP Selecta) and the fermentation bottles were inoculated with homogenized faecal material ( $10 \% w / v$ of fresh human faeces) for a period of $48 \mathrm{~h}$. After the addition of the lyophilized digested mango $(2 \mathrm{~g})$ the bottles were purged with 
oxygen-free nitrogen (OFN) and sealed airtight and the anaerobiotic conditions were maintained by using a continuous OFN flow. Aliquots $(1 \mathrm{~mL})$ of faecal suspensions were taken after $0,4,8,24$ and $48 \mathrm{~h}$. The samples were centrifuged at $13,500 \mathrm{rpm}$ at $4{ }^{\circ} \mathrm{C}$ for $10 \mathrm{~min}$ and stored immediately at $-80^{\circ} \mathrm{C}$ until analysis.

\subsection{Extraction of Polyphenols from Digested Mango Samples and from Faecal Incubates}

The extraction of polyphenols from in vitro digested mango samples and from faecal samples was adapted from Pereira-Caro et al. [25] and Alañón et al. [26] with some modifications. For the in vitro digested mango samples, $0.25 \mathrm{~g}$ of lyophilized sample was homogenized with $1 \mathrm{~mL}$ of a methanol/acidified water mixture $(80: 20, v / v)$ with $0.1 \%$ formic acid. The samples were centrifuged at $5000 \mathrm{rpm}$ for $10 \mathrm{~min}$ at $4{ }^{\circ} \mathrm{C}$, and supernatants were collected. The pellet was re-extracted with $1 \mathrm{~mL}$ of the same solvent as described above. All the supernatants were pooled to a final volume of $2 \mathrm{~mL}$. For the fermented mango samples, $0.5 \mathrm{~mL}$ of faecal incubates were extracted using $0.5 \mathrm{~mL}$ of $0.1 \%$ formic acid in methanol/acidified water $(80: 20, v / v)$, vortexed and centrifuged at $5000 \mathrm{rpm}$ for $10 \mathrm{~min}$ at $4{ }^{\circ} \mathrm{C}$, and supernatants were collected.

\subsection{UHPLC-HRMS Polyphenol Analysis}

Polyphenols extracted from the in vitro digested mango and faecal incubation samples were analyzed using an Ultimate 3000 RS UHPLC system (Dionex, San José, CA, USA) described previously in Moreno-Ortega et al. [23]. A Zorbax SB-C18 RRHD column $(100 \times 2.1 \mathrm{~mm}$ i.d., $1.8 \mu \mathrm{m}$ (Agilent, Santa Clara, CA, USA) preceded by a guard precolumn of the same stationary phase and maintained at $40{ }^{\circ} \mathrm{C}$ was ued for polyphenol HPLC separation. The flow rate was set to $0.2 \mathrm{~mL} / \mathrm{min}$ with a $26 \mathrm{~min}$ gradient of phase A: deionized water with $0.1 \%$ formic acid and B: acetonitrile with $0.1 \%$ formic acid. The gradient started at 3\% B, was maintained for $2 \mathrm{~min}$, then rose to $65 \% \mathrm{~B}$ in $18 \mathrm{~min}$, before rising to $80 \% \mathrm{~B}$ in $1 \mathrm{~min}$ and being maintained for $6 \mathrm{~min}$ with a 26 min gradient. Next, the column was equilibrated to the previous conditions within $10 \mathrm{~min}$. The Exactive Orbitrap mass spectrometer, fitted with a heated electrospray ionization probe (ThermoFisher Scientific, San José, CA, USA), operated in negative ionization mode (scanning from 100 to $1000 \mathrm{~m} / \mathrm{z}$ ). The capillary temperature and the heater temperature were set to $300^{\circ} \mathrm{C}$ and $150{ }^{\circ} \mathrm{C}$, respectively. 20 units were the sheath gas and the auxiliary gas flow rate, the sweep gas was 3 units, and the spray voltage was $4.00 \mathrm{kV}$. Xcalibur (3.0 software) was used for data acquisition and data processing.

The polyphenols in digested and fermented mango samples were identified by comparing the exact mass and the retention time with available commercially standards. In the absence of standards, polyphenols were putatively identified by comparing the theoretical exact mass of the molecular ion with its measured accurate mass, and referred to databases or libraries containing HRMS spectral information such as Phenol Explorer (http://phenol-explorer.eu/), Phytohub (http://phytohub.eu/) and Metlin (https://metlin.scripps.edu/landing_page.php?pgcontent=mainPage) databases. Identifications were categorized according to the annotation described by Summer et al. [27] using the MSIMI level. The phenolic compounds were quantified by selecting the theoretical exact mass of the molecular ion by reference to 0.01 to $100 \mathrm{ng} / \mu \mathrm{L}$ standard curves. The linearity was determined for all the commercially available standards. Limits of detection (LOD) and limits of quantification (LOQ) were from 0.01 to $0.2 \mathrm{ng}$ and from 0.05 to $0.8 \mathrm{ng}$, respectively. In absence of reference compounds, the polyphenols were quantified by reference to the calibration curve of a closely related parent compound.

\subsection{Bioaccessibility of (Poly)Phenols}

The percentage of bioaccessibility (bioaccessibility index) after simulated gastrointestinal digestion [23] was calculated as percentage of the final concentration of each compound after the simulated gastrointestinal digestion and the initial concentration of each compound in the non-digested samples. 


\subsection{Statistical Analysis}

Statistical analyses were carried out with three replicate measures of each sample. R software (v. 3.6.3, R Core Team, Vienna, Austria) was used to do the one-way ANOVA to determine significant differences between the different phases of the in vitro gastrointestinal digestion and the fecal fermentation. Then, the Tukey Honestly Significant Difference (HSD) post-hoc test was used for pairwise comparison.

\section{Results and Discussion}

\subsection{Polyphenols in Mango Pulp Samples}

A total of 43 polyphenol compounds belonging to different families were identified in the mango samples. Details of the UHPLC-HRMS characteristics, including the retention time (Rt), the experimental accurate mass and the error ( $\mathrm{ppm}$ ) between the exact accurate mass and the mass found of the detected compounds are summarized in Table 1.

Table 1. UHPLC-HRMS Characteristics of Polyphenol Compounds Identified and Quantified in Mango Samples. RT: retention time; $[\mathrm{M}-\mathrm{H}]^{-}$Exp: experimental exact mass; $\Delta$ : mass error.

\begin{tabular}{|c|c|c|c|c|c|}
\hline $\begin{array}{c}\mathrm{RT} \\
(\mathrm{min})\end{array}$ & Compound & $\begin{array}{l}\text { Chemical } \\
\text { Structure }\end{array}$ & $\begin{array}{c}{[\mathrm{M}-\mathrm{H}]^{-}} \\
\operatorname{Exp} \cdot(\mathrm{m} / \mathrm{z})^{-}\end{array}$ & $\begin{array}{c}\Delta \\
(\mathrm{ppm})\end{array}$ & MSIMI $^{\text {a }}$ \\
\hline & Phenolic Acid Derivatives & & & & \\
\hline 1.3 & Benzene-1,2-diol (pyrogallol) & $\mathrm{C}_{6} \mathrm{H}_{6} \mathrm{O}_{3}$ & 125.0233 & 0.39 & 1 \\
\hline 3.1 & 3,4,5-Triydroxybenzoic acid (gallic acid) & $\mathrm{C}_{7} \mathrm{H}_{6} \mathrm{O}_{5}$ & 169.0137 & 0.77 & 1 \\
\hline 6.6 & Gallic acid hexoside & $\mathrm{C}_{13} \mathrm{H}_{16} \mathrm{O}_{11}$ & 347.0608 & 1.73 & 2 \\
\hline 8.8 & 3-O-Methylgallic acid & $\mathrm{C}_{8} \mathrm{H}_{7} \mathrm{O}_{5}$ & 183.0287 & 1.8 & 1 \\
\hline 11.9 & 4-O-Methylgallic acid & $\mathrm{C}_{8} \mathrm{H}_{7} \mathrm{O}_{5}$ & 183.0287 & 1.8 & 1 \\
\hline 8.7 & 3,4-Dihydroxybenzoic acid & $\mathrm{C}_{7} \mathrm{H}_{6} \mathrm{O}_{4}$ & 153.0182 & 0.23 & 1 \\
\hline 9 & Benzoic acid & $\mathrm{C}_{7} \mathrm{H}_{6} \mathrm{O}_{2}$ & 121.0284 & 0.74 & 1 \\
\hline 4.7 & Hydroxybenzoic acid hexoside 1 & $\mathrm{C}_{13} \mathrm{H}_{16} \mathrm{O}_{8}$ & 299.0767 & 3.76 & 2 \\
\hline 7 & Hydroxybenzoic acid hexoside 2 & $\mathrm{C}_{13} \mathrm{H}_{16} \mathrm{O}_{8}$ & 299.0767 & 3.76 & 2 \\
\hline 7.4 & Syringic acid glucoside 1 & $\mathrm{C}_{15} \mathrm{H}_{20} \mathrm{O}_{10}$ & 359.0972 & 1.91 & 2 \\
\hline 7.8 & Syringic acid glucoside 2 & $\mathrm{C}_{15} \mathrm{H}_{20} \mathrm{O}_{10}$ & 359.0972 & 1.91 & 2 \\
\hline 8.8 & Methyl gallate & $\mathrm{C}_{8} \mathrm{H}_{8} \mathrm{O}_{5}$ & 183.0287 & 1.64 & 2 \\
\hline 11.9 & Methyl digallate ester 1 & $\mathrm{C}_{15} \mathrm{H}_{21} \mathrm{O}_{9}$ & 335.0397 & 4.3 & 2 \\
\hline 13 & Methyl digallate ester 2 & $\mathrm{C}_{15} \mathrm{H}_{21} \mathrm{O}_{9}$ & 335.0397 & 4.3 & 2 \\
\hline 7.1 & $\begin{array}{l}\text { 4-Hydroxy-3-methoxybenzaldehyde } \\
\text { (vanillic acid) }\end{array}$ & $\mathrm{C}_{8} \mathrm{H}_{8} \mathrm{O}_{4}$ & 167.0344 & 058 & 1 \\
\hline 3.8 & $\begin{array}{l}\text { Galloyl-quinic acid } \\
\text { Flavan-3-ol derivatives }\end{array}$ & $\mathrm{C}_{14} \mathrm{H}_{16} \mathrm{O}_{10}$ & 343.0659 & 1.63 & 2 \\
\hline 8.4 & Epigallocatechin & $\mathrm{C}_{15} \mathrm{H}_{14} \mathrm{O}_{7}$ & 305.0655 & 2.39 & 1 \\
\hline 9.8 & Epigallocatechin gallate & $\mathrm{C}_{22} \mathrm{H}_{18} \mathrm{O}_{11}$ & 457.0765 & 3.87 & 1 \\
\hline 11.1 & $\begin{array}{l}\text { Epicatechin gallate } \\
\text { Flavanone Derivatives }\end{array}$ & $\mathrm{C}_{22} \mathrm{H}_{18} \mathrm{O}_{11}$ & 457.0765 & 3.87 & 1 \\
\hline 8.8 & Eriodyctiol & $\mathrm{C}_{15} \mathrm{H}_{12} \mathrm{O}_{6}$ & 287.0550 & 3.22 & 1 \\
\hline 9.7 & Eriodyctiol hexoside 1 & $\mathrm{C}_{21} \mathrm{H}_{22} \mathrm{O}_{11}$ & 449.1078 & 1.59 & 2 \\
\hline 10.4 & Eriodyctiol hexoside 2 & $\mathrm{C}_{21} \mathrm{H}_{22} \mathrm{O}_{11}$ & 449.1078 & 1.59 & 2 \\
\hline 9.7 & Hesperetin glucoside 1 & $\mathrm{C}_{22} \mathrm{H}_{24} \mathrm{O}_{11}$ & 463.1234 & 0.18 & 2 \\
\hline 12.3 & $\begin{array}{l}\text { Hesperetin glucoside } 2 \\
\text { Flavonol Derivatives }\end{array}$ & $\mathrm{C}_{22} \mathrm{H}_{24} \mathrm{O}_{11}$ & 463.1234 & 0.18 & 2 \\
\hline 8.3 & Quercetin-hexoside 1 & $\mathrm{C}_{21} \mathrm{H}_{20} \mathrm{O}_{12}$ & 463.0877 & 2.09 & 2 \\
\hline 11.1 & Quercetin-hexoside 2 & $\mathrm{C}_{21} \mathrm{H}_{20} \mathrm{O}_{12}$ & 463.0877 & 2.09 & 2 \\
\hline 9.5 & Isorhamnetin hexoside 1 & $\mathrm{C}_{22} \mathrm{H}_{22} \mathrm{O}_{12}$ & 477.1027 & 2.05 & 2 \\
\hline 9.9 & Isorhamnetin hexoside 2 & $\mathrm{C}_{22} \mathrm{H}_{22} \mathrm{O}_{12}$ & 477.1027 & 2.05 & 2 \\
\hline
\end{tabular}


Table 1. Cont.

\begin{tabular}{|c|c|c|c|c|c|}
\hline $\begin{array}{c}\text { RT } \\
(\min )\end{array}$ & Compound & $\begin{array}{l}\text { Chemical } \\
\text { Structure }\end{array}$ & $\begin{array}{c}{[\mathrm{M}-\mathrm{H}]^{-}} \\
\operatorname{Exp}(\mathrm{m} / \mathrm{z})^{-}\end{array}$ & $\begin{array}{c}\Delta \\
(\mathrm{ppm})\end{array}$ & MSIMI $^{a}$ \\
\hline & Hydroxycinnamic acid derivatives & & & & \\
\hline 8.6 & Ferulic acid hexoside 1 & $\mathrm{C}_{16} \mathrm{H}_{20} \mathrm{O}_{9}$ & 355.1023 & 2.31 & 2 \\
\hline 9.5 & Ferulic acid hexoside 2 & $\mathrm{C}_{16} \mathrm{H}_{20} \mathrm{O}_{9}$ & 355.1023 & 2.31 & 2 \\
\hline 9 & Sinapic acid hexoside 1 & $\mathrm{C}_{17} \mathrm{H}_{22} \mathrm{O}_{10}$ & 385.1129 & 2.58 & 2 \\
\hline 9.3 & Sinapic acid hexoside 2 & $\mathrm{C}_{17} \mathrm{H}_{22} \mathrm{O}_{10}$ & 385.1129 & 2.58 & 2 \\
\hline 9.6 & sinapic acid hexoside 3 & $\mathrm{C}_{17} \mathrm{H}_{22} \mathrm{O}_{10}$ & 385.1129 & 2.58 & 2 \\
\hline 8 & Caffeoyl-hexoside 1 & $\mathrm{C}_{15} \mathrm{H}_{18} \mathrm{O}_{9}$ & 341.0867 & 2.53 & 2 \\
\hline 8.7 & Caffeoyl-hexoside 2 & $\mathrm{C}_{15} \mathrm{H}_{18} \mathrm{O}_{9}$ & 341.0867 & 2.53 & 2 \\
\hline 8.9 & Caffeoyl-quinic 1 & $\mathrm{C}_{16} \mathrm{H}_{18} \mathrm{O}_{9}$ & 353.0867 & 1.96 & 2 \\
\hline 10 & $\begin{array}{c}\text { Caffeoyl-quinic } 2 \\
\text { Xanthone }\end{array}$ & $\mathrm{C}_{16} \mathrm{H}_{18} \mathrm{O}_{9}$ & 353.0867 & 1.96 & 2 \\
\hline 9.4 & $\begin{array}{c}\text { Mangiferin } \\
\text { Galloyl derivatives }\end{array}$ & $\mathrm{C}_{19} \mathrm{H}_{18} \mathrm{O}_{11}$ & 421.0765 & 1.52 & 1 \\
\hline 2.7 & Mono-galloyl-glucose 1 & $\mathrm{C}_{13} \mathrm{H}_{16} \mathrm{O}_{10}$ & 331.0665 & 3.61 & 2 \\
\hline 6.6 & Mono-galloyl-glucose 2 & $\mathrm{C}_{13} \mathrm{H}_{16} \mathrm{O}_{10}$ & 331.0665 & 3.61 & 2 \\
\hline 9.9 & Tetra-O-galloyl glucoside 1 & $\mathrm{C}_{34} \mathrm{H}_{28} \mathrm{O}_{19}$ & 787.0994 & 2.04 & 2 \\
\hline 10.7 & Tetra-O-galloyl glucoside 2 & $\mathrm{C}_{34} \mathrm{H}_{28} \mathrm{O}_{19}$ & 787.0994 & 2.04 & 2 \\
\hline 11.3 & Penta-O-galloyl glucoside & $\mathrm{C}_{41} \mathrm{H}_{32} \mathrm{O}_{26}$ & 939.1035 & 1.87 & 2 \\
\hline
\end{tabular}

${ }^{\text {a }}$ Metabolite Standards Initiative Metabolite Identification (MSIMI) levels [26]. Reference compounds were available for all compounds identified at MSIMI level 1.

The following compounds were quantified: 16 phenolic acid derivatives, three flavan-3-ol derivatives, five flavanone derivatives, four flavonol derivatives, nine hydroxycinnamic acid derivatives, one xanthone and five gallotannin derivatives (Table 2). The main polyphenols were a hydroxybenzoic acid-hexoside (isomer 2) and two monogalloyl glucoside isomers, followed by the phenolic acid 4-hydroxy-3-methoxybenzaldehyde (vanillic acid), accounting for the $76.2 \%$ of the total polyphenols in the samples. Small quantities of other polyphenols were also detected, including pyrogallol ( $27 \mu \mathrm{mol} / \mathrm{g} D W)$, gallic acid $(46 \mu \mathrm{mol} / \mathrm{g} \mathrm{DW})$, gallic acid hexoside $(61 \mu \mathrm{mol} / \mathrm{g} \mathrm{DW})$, 3-O-methylgallic acid (47 $\mu \mathrm{mol} / \mathrm{g}$ DW), 4-O-methylgallic acid $(69 \mu \mathrm{mol} / \mathrm{g} \mathrm{DW})$, hydroxybenzoic acid hexoside $1(111 \mu \mathrm{mol} / \mathrm{g} D W)$, syringic acid hexoside $(2.7 \mu \mathrm{mol} / \mathrm{g} D W)$, methyl gallate $(1 \mu \mathrm{mol} / \mathrm{d} \mathrm{DW})$, methyl digallate ester (isomers 1 and 2) (16 and $1.6 \mu \mathrm{mol} / \mathrm{g}$ DW), two isomers of ferulic acid hexoside (48 and $63 \mu \mathrm{mol} / \mathrm{g}$ DW), three isomers of sinapic acid hexoside $(19,28$ and $35 \mu \mathrm{mol} / \mathrm{g} \mathrm{DW}$ ) and two caffeoyl-hexosides (47 and $3.3 \mu \mathrm{mol} / \mathrm{g}$ DW). Furthermore, the samples presented trace amounts of epicagallocatechin, epigallocatechin gallate, epicatechin gallate, eriodyctiol and two of its hexoside derivatives, hesperetin, quercetin, isorhamnetin and mangiferin. It is evident that the phytochemical composition of mango pulp is very complex, including several polyphenol families, making it difficult to compare their quantities with the literature as the composition is influenced by factors such as cultivar [15,28], storage conditions [29], ripening stage [26,30], and the part of the fruit analyzed, this being one of the factors with the greatest influence on the accumulation of bioactive compounds. For instance, the xanthone mangiferin, the flavonoids quercetin and kaempferol, and the phenolics gallic, ferulic, coumaric and caffeic acids have been shown to be the main polyphenols in mango leaves, peels and mango by-products [20,21] and in ethanolic extracts of peel and pulp residues [31]. In the case of the pulp, the phenolic acids gallic acid, caffeic acid, chlorogenic acid and protocatechuic acid were identified in different mango varieties, with gallic acid being predominant, followed by protocatechuic, chlorogenic, ferulic, vanillic and caffeic acids [32-35]. 
Table 2. Polyphenol compounds in mango puree samples before and after in vitro gastrointestinal digestion. Results are expressed as mean \pm standard deviation $(\mu \mathrm{mol} / \mathrm{g} \mathrm{DW})(n=3)$. BOD: before oral digestion; AOD: after oral digestion; AGD: after gastric digestion; AID: after intestinal digestion. \% Rem: \% remained; BI: Bioaccessibility Index.

\begin{tabular}{|c|c|c|c|c|c|c|c|c|}
\hline Compounds & BOD & AOD & $\%$ Rem & AGD & $\%$ Rem & AID & $B I$ & $p$-Value \\
\hline \multicolumn{9}{|l|}{ Phenolic acid derivatives } \\
\hline Benzene-1,2-diol (pyrogallol) & $27 \pm 3$ & $18 \pm 3$ & 66.7 & $21 \pm 9$ & 77.8 & $17 \pm 4$ & 63 & ns \\
\hline 3,4,5-Trihydroxybenzoic acid (gallic acid) & $46 \pm 4^{\mathrm{d}}$ & $109 \pm 16^{c}$ & 237 & $188 \pm 26^{b}$ & 408.7 & $267 \pm 36^{\mathrm{a}}$ & 580.4 & $* * *$ \\
\hline Gallic acid hexoside & $61 \pm 3^{a}$ & $49 \pm 9^{b}$ & 80.3 & $42 \pm 6^{b}$ & 68.9 & $22 \pm 3^{c}$ & 36.1 & $* * *$ \\
\hline 3-O-Methylgallic acid & $47 \pm 4^{b}$ & $86 \pm 8^{b}$ & 183 & $85 \pm 13^{b}$ & 180.9 & $2259 \pm 406^{a}$ & 4806 & $* * *$ \\
\hline 4-O-Methylgallic acid & $69 \pm 7^{c}$ & $132 \pm 28^{a}$ & 191.3 & $112 \pm 18^{b}$ & 162.3 & n.d. ${ }^{\mathrm{d}}$ & - & $* * *$ \\
\hline 3,4-Dihydroxybenzoic acid & n.d. & n.d. & & n.d. & & $176 \pm 39^{a}$ & 100 & $* * *$ \\
\hline Benzoic acid & n.d. & n.d. & & n.d. & & $84 \pm 15^{\mathrm{a}}$ & 100 & $* * *$ \\
\hline Hydroxybenzoic acid hexoside 1 & $111 \pm 45^{\mathrm{c}}$ & $434 \pm 88^{a b}$ & 391 & $472 \pm 141^{\mathrm{a}}$ & 425.2 & $322 \pm 110^{b}$ & 290.1 & $* * *$ \\
\hline Hydroxybenzoic acid hexoside 2 & $1259 \pm 327^{d}$ & $5644 \pm 571^{a}$ & 448.3 & $3985 \pm 444^{b}$ & 316.5 & $2287 \pm 208^{c}$ & 181.7 & $* * *$ \\
\hline Syringic acid glucoside 1 & $2.7 \pm 0.0^{\mathrm{a}}$ & $2.7 \pm 0.3^{\mathrm{a}}$ & 100 & $1.7 \pm 0.3^{\mathrm{b}}$ & 63 & $0.8 \pm 0.1^{\mathrm{c}}$ & 29.6 & $* * *$ \\
\hline Syringic acid glucoside 2 & $1.2 \pm 0.1^{\mathrm{a}}$ & $1.1 \pm 0.1^{b}$ & 91.7 & $0.5 \pm 0.1^{\mathrm{c}}$ & 41.7 & $0.1 \pm 0.0^{\mathrm{d}}$ & 8.3 & $* * *$ \\
\hline Methyl gallate & $1 \pm 1^{b}$ & $28 \pm 3^{b}$ & 2800 & $29 \pm 4^{b}$ & 2900 & $737 \pm 132^{a}$ & 737 & $* * *$ \\
\hline Methyl digallate ester 1 & $16 \pm 1^{b}$ & $30 \pm 6^{a}$ & 187.5 & $26 \pm 5^{a b}$ & 162.5 & $15 \pm 13^{b}$ & 93.8 & $*$ \\
\hline Methyl digallate ester 2 & $1.6 \pm 0.2^{b}$ & $2.4 \pm 0.7^{\mathrm{a}}$ & 150 & $2.5 \pm 0.5^{\mathrm{a}}$ & 156.3 & n.d. ${ }^{c}$ & - & $* * *$ \\
\hline $\begin{array}{l}\text { 4-Hydroxy-3-methoxybenzaldehyde } \\
\text { (vanillic acid) }\end{array}$ & $522 \pm 15^{a}$ & $486 \pm 60^{a}$ & 93.1 & $372 \pm 71^{b}$ & 7.3 & $158 \pm 48^{c}$ & 30.3 & $* * *$ \\
\hline Galloyl-quinic acid & $4.4 \pm 0.1^{\mathrm{a}}$ & $3.5 \pm 0.4^{b}$ & 79.5 & $3.3 \pm 0.4^{b}$ & 75 & $1.7 \pm 0.3^{c}$ & 38.6 & $* * *$ \\
\hline Total Phenolic Acid Derivatives & $2169 \pm 410^{c}$ & $7026 \pm 793^{a}$ & 323.9 & $5152 \pm 738^{b}$ & 246.2 & $6347 \pm 1014^{\mathrm{a}}$ & 292.6 & $* *$ \\
\hline \multicolumn{9}{|l|}{ Flavan-3-ol derivatives } \\
\hline Epigallocatechin & $37 \pm 0.5^{\mathrm{a}}$ & $38 \pm 3^{a}$ & 102.7 & $24 \pm 2^{b}$ & 64.9 & $18 \pm 2^{c}$ & 48.6 & $* * *$ \\
\hline Epigallocatechin gallate & $4.3 \pm 0.2^{\mathrm{a}}$ & $4.4 \pm 1.4^{\mathrm{a}}$ & 102.3 & $0.9 \pm 0.2^{b}$ & 20.9 & $0.00 \pm 0.00^{\mathrm{b}}$ & - & $* * *$ \\
\hline Epicatechin gallate & $0.21 \pm 0.02^{b}$ & $0.27 \pm 0.04^{\mathrm{a}}$ & 128.6 & $0.02 \pm 0.01^{\mathrm{c}}$ & 9.5 & $0.00 \pm 0.00^{c}$ & - & $* * *$ \\
\hline Total Flavan-3-ol derivatives & $41.5 \pm 0.7^{\mathrm{a}}$ & $42.7 \pm 4.4^{\mathrm{a}}$ & 102.8 & $24.9 \pm 2.2^{b}$ & 60 & $18 \pm 2^{c}$ & 43.4 & $* * *$ \\
\hline \multicolumn{9}{|l|}{ Flavanones Derivatives } \\
\hline Eriodyctiol & $0.66 \pm 0.04^{\mathrm{a}}$ & $0.68 \pm 0.09^{\mathrm{a}}$ & 103 & $0.27 \pm 0.05^{b}$ & 40.9 & n.d. ${ }^{c}$ & - & $* * *$ \\
\hline Eriodyctiol hexoside 1 & $0.11 \pm 0.01^{\mathrm{a}}$ & $0.09 \pm 0.03^{a}$ & 81.8 & $0.03 \pm 0.01 \mathrm{~b}$ & 27.3 & n.d. ${ }^{c}$ & - & $* * *$ \\
\hline Eriodyctiol hexoside 2 & $0.04 \pm 0.02^{a b}$ & $0.05 \pm 0.02^{\mathrm{a}}$ & 125 & $0.02 \pm 0.01^{b}$ & 50 & n.d. ${ }^{c}$ & - & $* * *$ \\
\hline Hesperetin glucoside 1 & $0.12 \pm 0.05^{\mathrm{a}}$ & $0.17 \pm 0.07^{\mathrm{a}}$ & 141.7 & n.d. ${ }^{b}$ & 0.0 & n.d. $b$ & - & $* * *$ \\
\hline
\end{tabular}


Table 2. Cont.

\begin{tabular}{|c|c|c|c|c|c|c|c|c|}
\hline Compounds & BOD & AOD & \% Rem & AGD & $\%$ Rem & AID & $B I$ & $p$-Value \\
\hline Hesperetin glucoside 2 & $0.03 \pm 0.00^{\mathrm{a}}$ & n.d. ${ }^{b}$ & 0 & n.d. ${ }^{b}$ & 0.0 & n.d. $b$ & - & $* * *$ \\
\hline \multicolumn{9}{|l|}{ Flavonols Derivatives } \\
\hline Quercetin-hexoside 1 & $0.03 \pm 0.01^{\mathrm{a}}$ & $0.02 \pm 0.01^{b}$ & 66.7 & $0.02 \pm 0.01^{b}$ & 66.7 & n.d. ${ }^{c}$ & - & $* *$ \\
\hline Quercetin-hexoside 2 & $0.10 \pm 0.00^{\mathrm{a}}$ & $0.10 \pm 0.01^{\mathrm{a}}$ & 100 & $0.06 \pm 0.01^{b}$ & 60 & $0.02 \pm 0.01^{\mathrm{c}}$ & 20 & $* * *$ \\
\hline Isorhamnetin hexoside 1 & $0.01 \pm 0.00^{\mathrm{a}}$ & $0.01 \pm 0.00 \mathrm{a}$ & 100 & n.d. b & 0 & n.d. $b$ & - & ** \\
\hline Isorhamnetin hexoside 2 & $0.70 \pm 0.01^{\mathrm{a}}$ & $0.67 \pm 0.07^{\mathrm{a}}$ & 95.7 & $0.50 \pm 0.07^{b}$ & 71.4 & $0.37 \pm 0.07^{c}$ & 52.9 & $* * *$ \\
\hline Total flavonol derivatives & $0.84 \pm 0.02^{\mathrm{a}}$ & $0.80 \pm 0.09^{a}$ & 95.2 & $0.58 \pm 0.10^{b}$ & 69 & $0.39 \pm 0.08^{c}$ & 46.4 & ** \\
\hline \multicolumn{9}{|l|}{ Hydroxycinnamic acid derivatives } \\
\hline Ferulic acid hexoside 1 & $48 \pm 2^{a}$ & $48 \pm 4^{\mathrm{a}}$ & 100 & $27 \pm 3^{b}$ & 56.3 & $20 \pm 3^{c}$ & 41.7 & $* * *$ \\
\hline Ferulic acid hexoside 2 & $63 \pm 3^{a}$ & $70 \pm 8^{a}$ & 111.1 & $37 \pm 3^{b}$ & 58.7 & $13 \pm 2^{c}$ & 20.6 & $* * *$ \\
\hline Sinapic acid hexoside 1 & $19 \pm 2^{a}$ & $20 \pm 1^{\mathrm{a}}$ & 105.3 & $11 \pm 1^{b}$ & 57.9 & $7 \pm 2^{c}$ & 36.8 & $* * *$ \\
\hline Sinapic acid hexoside 2 & $28 \pm 2^{a}$ & $31 \pm 4^{\mathrm{a}}$ & 110.7 & $20 \pm 3^{b}$ & 71.4 & $11 \pm 1^{c}$ & 39.3 & $* * *$ \\
\hline sinapic acid hexoside 3 & $35 \pm 2^{a}$ & $35 \pm 4^{\mathrm{a}}$ & 100 & $19 \pm 2^{b}$ & 54.3 & $8 \pm 2^{c}$ & 22.0 & $* * *$ \\
\hline Caffeoyl-hexoside 1 & $47 \pm 2^{a}$ & $40 \pm 4^{b}$ & 85.1 & $17 \pm 3^{c}$ & 36.2 & $7.9 \pm 0.8^{\mathrm{d}}$ & 16.8 & $* * *$ \\
\hline Caffeoyl-hexoside 2 & $3.3 \pm 0.6^{\mathrm{a}}$ & $3.8 \pm 0.9^{a}$ & 115.2 & $1.6 \pm 0.3^{b}$ & 48.5 & $0.6 \pm 0.3^{c}$ & 18.2 & $* * *$ \\
\hline Caffeoyl-quinic 1 & $0.5 \pm 0.3^{\mathrm{a}}$ & $0.4 \pm 0.1^{\mathrm{a}}$ & 80 & n.d. b & 0 & n.d. ${ }^{b}$ & - & $* * *$ \\
\hline Caffeoyl-quinic 2 & $2.3 \pm 0.3^{a}$ & $2.5 \pm 0.2^{\mathrm{a}}$ & 108.7 & $1.09 \pm 0.19^{b}$ & 47.4 & $0.02 \pm 0.05^{c}$ & - & $* * *$ \\
\hline $\begin{array}{l}\text { Total Hydroxycinnamic acid derivatives } \\
\text { Xanthone }\end{array}$ & $246.0 \pm 14.2^{a}$ & $251 \pm 26^{a}$ & 101.9 & $133.7 \pm 15.5^{b}$ & 54.3 & $67.5 \pm 11.1^{\mathrm{c}}$ & 27.4 & $* * *$ \\
\hline $\begin{array}{c}\text { Mangiferin } \\
\text { Gallotannins derivatives }\end{array}$ & $0.12 \pm 0.01^{\mathrm{ab}}$ & $0.14 \pm 0.03^{a}$ & 116.7 & $0.09 \pm 0.02^{b}$ & 75 & $0.10 \pm 0.02^{b}$ & 83.3 & ** \\
\hline Mono-galloyl-glucose & $481 \pm 16^{\mathrm{a}}$ & $453 \pm 30^{\mathrm{a}}$ & 94.2 & $385 \pm 30^{b}$ & 80.0 & $516 \pm 85^{a}$ & 107.3 & $* *$ \\
\hline Mono-galloyl-glucose & $526 \pm 34^{a}$ & $465 \pm 53^{b}$ & 88.4 & $373 \pm 49^{c}$ & 70.9 & $197 \pm 21^{d}$ & 37.5 & $* * *$ \\
\hline Tetra-O-galloyl glucoside & $0.15 \pm 0.01^{c}$ & $0.33 \pm 0.06^{b}$ & 220 & $0.5 \pm 0.2^{\mathrm{a}}$ & 333.3 & $0.37 \pm 0.09^{b}$ & 246.7 & $* * *$ \\
\hline Tetra-O-galloyl glucoside & $1.72 \pm 0.08^{c}$ & $2.3 \pm 0.3^{b}$ & 133.7 & $2.7 \pm 0.4^{\mathrm{a}}$ & 157 & $2.2 \pm 0.4 b c$ & 127.9 & $*$ \\
\hline Penta-O-galloyl glucoside & $1.9 \pm 0.6^{\mathrm{b}}$ & $2.0 \pm 0.7^{b}$ & 107.9 & $2.0 \pm 0.4^{b}$ & 105.3 & $8 \pm 2^{a}$ & 421.1 & $* * *$ \\
\hline Total Galloyl derivatives & $1011 \pm 51^{\mathrm{a}}$ & $923 \pm 84^{b}$ & 91.3 & $763 \pm 80^{c}$ & 75.5 & $724 \pm 108^{c}$ & 71.6 & $* *$ \\
\hline TOTAL POLYPHENOLS & $3469 \pm 476^{d}$ & $8244 \pm 908^{a b}$ & 237.6 & $6075 \pm 836^{c}$ & 180.5 & $7156 \pm 1135^{b c}$ & 206.3 & $* *$ \\
\hline
\end{tabular}

Different letters within each row indicate significant difference among samples Ns: non-significant; ${ }^{*} p$-value $<0.05 ;{ }^{* *} p$-value $<0.01$; ${ }^{* * *} p$-value $<0.001$. n.d.: not detected. 


\subsection{Stability and Bioaccessibility of Phenolic Compounds in Mango after Simulated Gastrointestinal Digestion}

Table 2 shows the impact of in vitro gastrointestinal digestion on mango polyphenols. After in vitro oral digestion, the total content of polyphenols in the mango significantly increased (from 3.47 to $8.2 \mathrm{mmol} / \mathrm{g} \mathrm{DW}$ ), with a mean recovery of $237.6 \%$. The compounds mostly affected during this oral phase were the phenolic acid derivatives and the galloyl-derivatives.

The phenolic acids significantly increased their concentration from $2169 \mu \mathrm{mol} / \mathrm{g}$ DW to $7026 \mu \mathrm{mol} / \mathrm{g} \mathrm{DW}$, accounting for a mean recovery of $323.9 \%$, mainly due to the substantial increase of individual phenolics including gallic acid (from 46 to $109 \mu \mathrm{mol} / \mathrm{g} \mathrm{DW}$ ), 4-O-methylgallic acid (from 69 to $132 \mu \mathrm{mol} / \mathrm{g} \mathrm{DW}$ ), the two hydroxybenzoic acid hexoside isomers (from 1370 to $6078 \mu \mathrm{mol} / \mathrm{g} \mathrm{DW}$ ), methyl gallate (from 1 to $28 \mu \mathrm{mol} / \mathrm{g} \mathrm{DW}$ ) and the two methyl-gallate ester isomers (from 17.6 to $32.4 \mu \mathrm{mol} / \mathrm{g} \mathrm{DW})$. Meanwhile the galloyl-derivatives presented a slight decrease in concentration from 1010 to $923 \mu \mathrm{mol} / \mathrm{g}$ DW, showing a recovery of $91.3 \%$ after the oral phase, likely due to the decrease in the mono-galloyl glucoside isomers.

The remaining compounds in the mango, belonging to flavan-3-ol, flavanones, hydroxycinnamic acid and xanthone families, were stable under the oral digestion conditions, presenting mean recovery rates between $95.2 \%$ for flavonol derivatives and $116.7 \%$ for mangiferin. The increase in gallic acid and hydroxybenzoic acid hexoside during the oral phase is likely due to enzymatic activity and the $\mathrm{pH}$, which could induce the breakdown of these compounds from other food constituents, as previously reported by other authors in soy milk [36] and persimmon fruits [37].

After in vitro gastric digestion, the total content of polyphenols in mango pulp significantly decreased compared to those obtained after oral digestion (from 8.2 to $6.07 \mathrm{mmol} / \mathrm{g} \mathrm{DW}$ ), but the total concentration was still higher than the initial quantities of polyphenols in the mango pulp. Despite the notable decrease in the polyphenol content after gastric digestion of almost all the polyphenol classes-losses ranging from $100 \%$ for hesperetin glucosides isomers to $20 \%$ for monogalloyl-glucoside - there were some specific compounds that presented significant increases in their concentration under the gastric conditions. Specifically, as likely occurred during the oral phase, the phenolic acids, namely gallic acid, the two hydroxybenzoic acid hexoside isomers, methyl gallate and the two methyl-gallate ester isomers, were positively influenced by the gastric conditions and substantially increased their concentrations after in vitro gastric digestion. Recovery rates ranged between 156.3 and $2900 \%$, while the two tetra-galloyl glucoside isomers presented recoveries of 333.3 and $157 \%$, respectively. These changes can be explained taking into consideration that during the gastric phase, the polyphenols, which are hydrogen bonding structures or are covalently linked to cell wall polysaccharides in the food matrix, can be released due to the low $\mathrm{pH}$ and pepsin activity [38]. In keeping with our results, Lucas-González et al. [37] found a significant increase in gallic acid content in persimmon fruits after the gastric phase.

After the in vitro intestinal digestion, the total polyphenol content in the mango pulp continue increasing, from 6.07 to $7.15 \mathrm{mmol} / \mathrm{g}$ DW, with a mean bioaccessibility index of $206.3 \%$. This increase was mostly due to the huge increase in the concentration of specific phenolic compounds after the intestinal digestion, such as gallic acid (from 188 to $267 \mu \mathrm{mol} / \mathrm{g} \mathrm{DW}$ ), 3-O-methylgallic acid (from 85 to $2259 \mu \mathrm{mol} / \mathrm{g} \mathrm{DW}$ ), methyl gallate (from 29 to $737 \mu \mathrm{mol} / \mathrm{g} \mathrm{DW}$ ), in addition to the appearance of 3,4-dihydroxybenzoic acid $(176 \mu \mathrm{mol} / \mathrm{g} \mathrm{DW})$ and benzoic acid $(84 \mu \mathrm{mol} / \mathrm{g} \mathrm{DW})$, arguably from the hydrolysis of monogallyl glucoside and hydroxybenzoic acid hexoside, respectively. At intestinal level, factors such as $\mathrm{pH}$ and the action of the enzymes (pancreatin) and bile salts could favor the breakage of the weak bond between the supramolecular structures of the food matrix and the polyphenols, mainly the ones with low molecular weight, releasing them from the food matrix during the digestion and, therefore, increasing their bioaccessibility [21]. All these compounds are potentially available for absorption at the end of intestinal digestion. Moreover, despite the increase in the total quantities of polyphenols after in vitro digestion, the remaining polyphenols, including the flavan-3-ols, flavanols, hydroxycinnamic acid derivatives and xanthone, were negatively affected by the digestive process and their concentration significantly decreased during the digestive process. The losses ranged from 
$6.2 \%$ for methyl digallate ester 1 to completely disappearing, as in the case of 4-O-methylgallic acid, methyl-digallate ester (isomer 2), epigallocatechin gallate, epicatechin gallate, eriodictyol hexoside 1 and 2, hesperetin-glucoside (1 and 2), quercetin hexoside 1, isorhamnetin hexoside 1 and caffeoyl-quinic acid 1. Overall, the most bioaccessible compounds of the mango pulp were gallic acid, 3-O-methylgallic acid, the two hydroxybenzoic acid hexosides, methyl gallate, 3,4-dihydroxybenzoic acid and benzoic acid, which potentially cross the small intestine and reach the colon for fermentation by the resident microbiota. These results are in keeping with those reported by Blancas-Benitez et al. [18], who also found that the major polyphenols released during the in vitro digestion process of mango paste were gallic acid and hydroxybenzoic acids.

\subsection{Degradation of Mango Polyphenols during Fecal Fermentation}

After the in vitro digestion process, which mimics the steps that would occur in vivo before the food enters the large intestine to be fermented, the digested mango pulp samples were incubated with a fresh and homogenized fecal suspension from three donors for a period of $48 \mathrm{~h}$ under anaerobic conditions and analyzed by HPLC-HRMS. Details of the UHPLC-HRMS characteristics of the detected microbial-derived compounds are shown in Table 3.

Table 3. UHPLC-HRMS Characteristics of Mango Polyphenol Catabolites Identified after fecal fermentation.

\begin{tabular}{cccccc}
\hline $\begin{array}{c}\text { RT } \\
(\mathbf{m i n})\end{array}$ & Catabolites & $\begin{array}{c}\text { Chemical } \\
\text { Structure }\end{array}$ & $\begin{array}{c}{[\mathbf{M}-\mathbf{H}]^{-}} \\
\text {Exp. }(\mathbf{m} / \mathbf{z})\end{array}$ & $\begin{array}{c}\boldsymbol{\Delta} \\
(\mathbf{p p m})\end{array}$ & MSIMI $^{\mathbf{a}}$ \\
\hline 10.6 & 3-(4-Hydroxyphenyl)propanoic acid & $\mathrm{C}_{9} \mathrm{H}_{10} \mathrm{O}_{3}$ & 165.0546 & 1.54 & 1 \\
7.9 & 3-Phenylacetic acid & $\mathrm{C}_{8} \mathrm{H}_{8} \mathrm{O}_{2}$ & 135.0440 & 0.84 & 1 \\
8.8 & (-)-Epicatechin & $\mathrm{C}_{15} \mathrm{H}_{14} \mathrm{O}_{6}$ & 289.0706 & 0.14 & 1 \\
10.4 & Norathyriol & $\mathrm{C}_{13} \mathrm{H}_{8} \mathrm{O}_{6}$ & 259.0216 & -2.07 & 2 \\
\hline
\end{tabular}

a Metabolite Standards Initiative Metabolite Identification (MSIMI) levels [27]. Reference compounds were available for all compounds identified at MSIMI level 1.

The results from the in vitro fecal fermentation, presented in Table 4, revealed that the human microbiota gradually converted the mango polyphenols remaining after the in vitro gastrointestinal digestion. The overall quantities of mango polyphenols and their catabolites present after 4, 8, 24 and $48 \mathrm{~h}$ of fermentation were 4095, 4202, 2286 and $2043 \mu \mathrm{mol} / \mathrm{g}$ DW, respectively, corresponding to 101, 103, 56 and $50 \%$ of the initial amounts (Table 4). The major end products were pyrogallol, gallic acid and 3,4-dihydroxybenzoic acid, comprising $97.4 \%$ of the total catabolites; the remaining $2.5 \%$ comprised the minor catabolites such as hydroxybenzoic acid hexoside 2, 3-(4'-dihydroxy-phenyl)propanoic acid, 3-phenylacetic acid, (-)-epicatechin, eriodictyol, caffeoyl hexoside 1 and the monogalloyl hexoside 1 (Table 4).

After an incubation time of $4 \mathrm{~h}$, significant quantities of pyrogallol $(40 \mu \mathrm{mol} / \mathrm{g} \mathrm{DW})$, gallic acid $(410 \mu \mathrm{mol} / \mathrm{g} \mathrm{DW})$ and 3,4-dihydroxybenzoic acid $(949 \mu \mathrm{mol} / \mathrm{g} \mathrm{DW})$ were detected, attaining the highest accumulation of these compounds after incubation periods of 48,24 and $8 \mathrm{~h}$, respectively

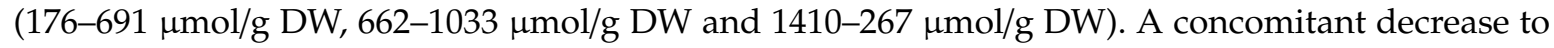
almost zero of the mono-galloyl-glucosides and hydroxybenzoic acid-hexosides was also evidenced after $48 \mathrm{~h}$ of incubation (Table 4, Figure 1A). These data indicate that a plausible mechanism for galloyl-glucoside degradation by human microbiota is, first, the microbial-mediated transformation of mono-galloyl-glucoside into gallic acid, as the first catabolic event, which is further decarboxylated or dehydroxylated producing pyrogallol and 3,4-dihydroxybenzoic acid, as proposed in Figure 1B. In line with our results, Hernandez-Maldonado et al. [20] reported that gallic acid was identified during the in vitro fermentation of digested mango bars, the maximum amount being reached after $6 \mathrm{~h}$ of incubation. Moreover, gallic acid has been identified in human urine after mango pulp consumption $[39,40]$ and was inversely correlated with the urinary excretion of pyrogallol, indicating the colonic origin of this catabolite. 
Table 4. Stability of native mango polyphenols and catabolites produced during $0,4,8,24$ and $48 \mathrm{~h}$ of faecal fermentation. Results are expressed as mean \pm standard deviation ( $\mu \mathrm{mol} / \mathrm{g} \mathrm{DW})(n=3)$.

\begin{tabular}{|c|c|c|c|c|c|c|c|c|c|c|c|}
\hline \multirow{2}{*}{ Compounds } & \multicolumn{2}{|r|}{$0 \mathrm{~h}$} & \multicolumn{2}{|c|}{$4 \mathrm{~h}$} & \multicolumn{2}{|c|}{$8 \mathrm{~h}$} & \multicolumn{2}{|c|}{$24 \mathrm{~h}$} & \multicolumn{2}{|r|}{$48 \mathrm{~h}$} & \multirow{2}{*}{$p$-Value } \\
\hline & Control & Mango & Control & Mango & Control & Mango & Control & Mango & Control & Mango & \\
\hline \multicolumn{12}{|c|}{ Phenolic acid derivatives } \\
\hline Benzene-1,2-diol (pyrogallol) & n.d. ${ }^{f}$ & $17 \pm 4^{\mathrm{e}}$ & n.d. ${ }^{f}$ & $40 \pm 2^{d}$ & n.d. ${ }^{f}$ & $176 \pm 5^{c}$ & n.d. ${ }^{f}$ & $465 \pm 10^{b}$ & n.d. ${ }^{f}$ & $691 \pm 49^{a}$ & $* * *$ \\
\hline 3,4,5-Triydroxybenzoic acid (gallic acid) & n.d. e & $268 \pm 36^{d}$ & n.d. ${ }^{e}$ & $410 \pm 11^{\mathrm{c}}$ & n.d. ${ }^{e}$ & $662 \pm 61^{b}$ & n.d. ${ }^{e}$ & $1092 \pm 61^{\mathrm{a}}$ & n.d. ${ }^{e}$ & $1033 \pm 38^{\mathrm{a}}$ & $* * *$ \\
\hline Gallic acid hexoside & n.d. ${ }^{c}$ & $21.9 \pm 0.9^{\mathrm{a}}$ & n.d. ${ }^{c}$ & $1.8 \pm 0.1^{b}$ & n.d. ${ }^{c}$ & $1.1 \pm 0.1^{b}$ & n.d. ${ }^{c}$ & n.d. ${ }^{c}$ & n.d. ${ }^{c}$ & n.d. ${ }^{c}$ & $* * *$ \\
\hline 3,4-Dihydroxybenzoic acid & n.d. ${ }^{\mathrm{f}}$ & $177 \pm 39^{\mathrm{e}}$ & n.d. ${ }^{f}$ & $949 \pm 108^{b}$ & n.d. $\mathrm{f}$ & $1410 \pm 85^{\mathrm{a}}$ & n.d. ${ }^{\mathrm{f}}$ & $659 \pm 184^{c}$ & n.d. ${ }^{f}$ & $267 \pm 28^{d}$ & *** \\
\hline Hydroxybenzoic acid hexoside 1 & n.d. ${ }^{c}$ & $323 \pm 56^{a}$ & n.d. ${ }^{c}$ & $338 \pm 5^{\mathrm{a}}$ & n.d. ${ }^{c}$ & $217 \pm 52^{b}$ & n.d. ${ }^{c}$ & n.d. ${ }^{c}$ & n.d. ${ }^{c}$ & n.d. ${ }^{c}$ & $* * *$ \\
\hline Hydroxybenzoic acid hexoside 2 & n.d. ${ }^{d}$ & $2294 \pm 102^{a}$ & n.d. ${ }^{d}$ & $1569 \pm 36^{\mathrm{b}}$ & n.d. ${ }^{d}$ & $1080 \pm 68^{c}$ & n.d. d & $41 \pm 13^{\mathrm{d}}$ & n.d. d & $27 \pm 2^{d}$ & $* * *$ \\
\hline Syringic acid glucoside 1 & n.d. ${ }^{c}$ & $0.9 \pm 0.1^{\mathrm{a}}$ & n.d. ${ }^{c}$ & $0.7 \pm 0.0^{b}$ & n.d. ${ }^{c}$ & $0.7 \pm 0.1^{b}$ & n.d. ${ }^{c}$ & n.d. ${ }^{c}$ & n.d. ${ }^{c}$ & n.d. ${ }^{c}$ & $* * *$ \\
\hline Syringic acid glucoside 2 & n.d. ${ }^{b}$ & $0.2 \pm 0.0^{\mathrm{a}}$ & n.d. ${ }^{b}$ & $0.1 \pm 0.0^{\mathrm{a}}$ & n.d. ${ }^{b}$ & n.d. ${ }^{b}$ & n.d. ${ }^{b}$ & n.d. ${ }^{b}$ & n.d. ${ }^{b}$ & n.d. ${ }^{b}$ & $* * *$ \\
\hline $\begin{array}{l}\text { 4-Hydroxy-3-methoxybenzaldehyde } \\
\text { (vanillic acid) }\end{array}$ & n.d. ${ }^{c}$ & $162 \pm 14^{\mathrm{a}}$ & n.d. ${ }^{c}$ & $104 \pm 44^{b}$ & n.d. ${ }^{c}$ & $167 \pm 12^{\mathrm{a}}$ & n.d. ${ }^{c}$ & n.d. ${ }^{c}$ & n.d. ${ }^{c}$ & n.d. ${ }^{c}$ & $* * *$ \\
\hline 3-(4-Hydroxyphenyl)propanoic acid & n.d. ${ }^{d}$ & n.d. ${ }^{d}$ & $0.9 \pm 0.1$ & $2 \pm 1^{c}$ & $0.4 \pm 0.2^{\mathrm{c}}$ & $9 \pm 1^{b}$ & $0.4 \pm 0.1$ & $18 \pm 3^{\mathrm{a}}$ & n.d. ${ }^{e}$ & $19 \pm 1^{\mathrm{a}}$ & $* * *$ \\
\hline 3-Phenylacetic acid & n.d. ${ }^{b}$ & n.d. ${ }^{b}$ & n.d. ${ }^{b}$ & $\begin{array}{l}\text { n.d. }{ }^{b} \\
\text { Flavan-3-ol c }\end{array}$ & $\begin{array}{c}\text { n.d. } \\
\text { erivatives }\end{array}$ & n.d. ${ }^{b}$ & n.d. ${ }^{b}$ & $26 \pm 11^{a}$ & n.d. ${ }^{b}$ & $41 \pm 8^{\text {a }}$ & $* * *$ \\
\hline $\begin{array}{l}\text { Epigallocatechin } \\
\text { (-)-Epicatechin }\end{array}$ & $\begin{array}{l}\text { n.d. }{ }^{f} \\
\text { n.d. }\end{array}$ & $\begin{array}{l}21 \pm 1^{\text {a }} \\
\text { n.d. }\end{array}$ & $\begin{array}{l}\text { n.d. }{ }^{f} \\
\text { n.d. }\end{array}$ & $\begin{array}{c}13 \pm 1^{\mathrm{b}} \\
8.2 \pm 0.3^{\mathrm{a}}\end{array}$ & $\begin{array}{l}\text { n.d. }{ }^{f} \\
\text { n.d. }\end{array}$ & $\begin{array}{l}5.8 \pm 0.8^{c} \\
7.5 \pm 0.0^{a}\end{array}$ & $\begin{array}{l}\text { n.d. }{ }^{f} \\
\text { n.d. }\end{array}$ & $\begin{array}{l}1.3 \pm 0.1^{\mathrm{e}} \\
5.7 \pm 0.0^{\mathrm{b}}\end{array}$ & $\begin{array}{l}\text { n.d. }{ }^{f} \\
\text { n.d. }\end{array}$ & $\begin{array}{l}1.5 \pm 0.1^{\mathrm{e}} \\
2.7 \pm 0.2^{\mathrm{c}}\end{array}$ & $* * *$ \\
\hline \multicolumn{12}{|c|}{ Hydroxycinnamic acid derivatives } \\
\hline Ferulic acid hexoside 1 & n.d. ${ }^{c}$ & $18 \pm 2^{a}$ & n.d. ${ }^{c}$ & $10.9 \pm 0.8^{\mathrm{b}}$ & n.d. ${ }^{c}$ & n.d. ${ }^{c}$ & n.d. ${ }^{c}$ & n.d. ${ }^{c}$ & n.d. ${ }^{c}$ & n.d. ${ }^{c}$ & $* * *$ \\
\hline Sinapic acid hexoside 1 & n.d. d & $7 \pm 1^{\mathrm{c}}$ & n.d. d & $19 \pm 1^{b}$ & n.d. d & $35 \pm 3^{a}$ & n.d. ${ }^{d}$ & n.d. ${ }^{d}$ & n.d. d & n.d. d & $* * *$ \\
\hline Caffeoyl-hexoside 1 & n.d. ${ }^{c}$ & $8 \pm 1^{\mathrm{a}}$ & n.d. ${ }^{c}$ & $2.8 \pm 0.4^{b}$ & n.d. ${ }^{c}$ & $3.1 \pm 0.3^{b}$ & n.d. ${ }^{c}$ & $1.4 \pm 0.1^{b}$ & n.d. c & $1.4 \pm 0.2^{b}$ & $* * *$ \\
\hline Caffeoyl-hexoside 2 & n.d. ${ }^{c}$ & $0.6 \pm 0.1^{b}$ & n.d. ${ }^{c}$ & $2.0 \pm 0.0^{\mathrm{a}}$ & n.d. ${ }^{c}$ & $2.6 \pm 0.1^{\mathrm{a}}$ & n.d. ${ }^{c}$ & n.d. ${ }^{c}$ & n.d. ${ }^{c}$ & n.d. ${ }^{c}$ & $* * *$ \\
\hline $\begin{array}{l}\text { Mangiferin } \\
\text { Norathyriol }\end{array}$ & $\begin{array}{l}\text { n.d. }{ }^{b} \\
\text { n.d. }\end{array}$ & $\begin{array}{l}0.10 \pm 0.01^{a} \\
\text { n.d. }{ }^{c}\end{array}$ & $\begin{array}{l}\text { n.d. }{ }^{b} \\
\text { n.d. }\end{array}$ & $\begin{array}{l}\text { Xanthone d } \\
\text { n.d. } \mathrm{b} \\
0.07 \pm 0.02 \text { a } \\
\text { Galloyl de }\end{array}$ & $\begin{array}{l}\text { rivatives } \\
\text { n.d. }{ }^{b} \\
\text { n.d. }{ }^{c} \\
\text { vatives }\end{array}$ & $\begin{array}{c}\text { n.d. }^{\mathrm{b}} \\
0.02 \pm 0.01^{\mathrm{b}}\end{array}$ & $\begin{array}{l}\text { n.d. }{ }^{b} \\
\text { n.d. }{ }^{c}\end{array}$ & $\begin{array}{c}\text { n.d. }{ }^{b} \\
0.02 \pm 0.01 b\end{array}$ & $\begin{array}{l}\text { n.d. }{ }^{b} \\
\text { n.d. }\end{array}$ & $\begin{array}{l}\text { n.d. }{ }^{b} \\
\text { n.d. }\end{array}$ & $* * * *$ \\
\hline Mono-galloyl-glucose 1 & n.d. ${ }^{e}$ & $518 \pm 16^{\mathrm{a}}$ & n.d. ${ }^{e}$ & $384 \pm 25^{b}$ & n.d. $e^{e}$ & $266 \pm 42^{c}$ & n.d. ${ }^{e}$ & $19 \pm 2^{d}$ & n.d. ${ }^{e}$ & $17 \pm 5^{d}$ & $* * *$ \\
\hline Mono-galloyl-glucose 2 & n.d. ${ }^{c}$ & $220 \pm 34^{\mathrm{a}}$ & n.d. ${ }^{c}$ & $242.5 \pm 0.5^{\mathrm{a}}$ & n.d. ${ }^{c}$ & $173 \pm 32^{b}$ & n.d. ${ }^{c}$ & n.d. ${ }^{c}$ & n.d. ${ }^{c}$ & n.d. ${ }^{c}$ & $* * *$ \\
\hline Total phenolic compounds & n.d. ${ }^{c}$ & $4057 \pm 310^{\mathrm{a}}$ & $0.9 \pm 0.1^{\mathrm{g}}$ & $4095 \pm 239^{a}$ & $0.4 \pm 0.1^{\mathrm{f}}$ & $4202 \pm 364^{a}$ & $0.4 \pm 0.1^{f}$ & $2286 \pm 288^{b}$ & n.d. ${ }^{c}$ & $2043 \pm 135^{b}$ & $* * *$ \\
\hline
\end{tabular}

Different letters within each row indicate significant difference among samples. ${ }^{* * *} p$-value $<0.001$. n.d.: not detected. 


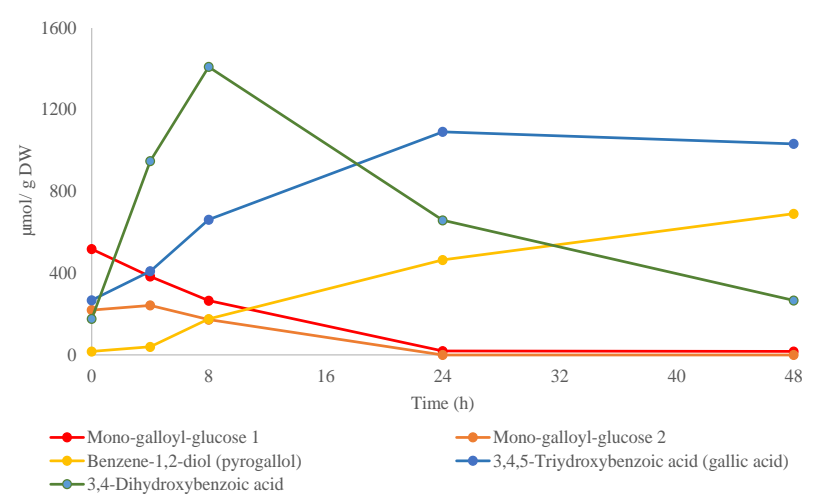

(A)
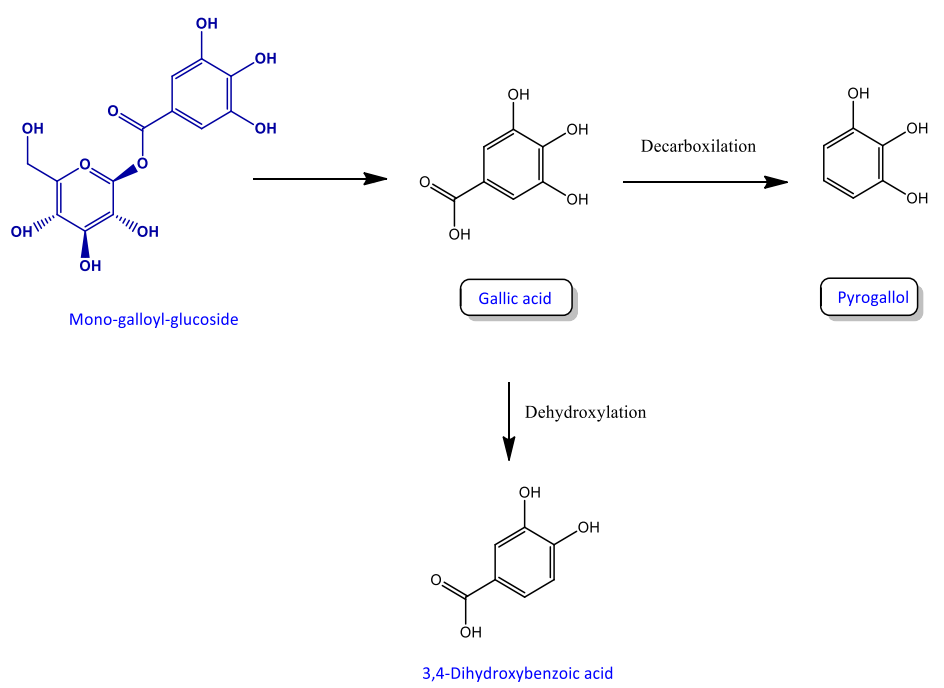

(B)

Figure 1. (A) Monogalloyl hexoside degradation profiles over $48 \mathrm{~h}$ of incubation with human faeces. Data expressed as $\mu \mathrm{mol} \pm \mathrm{SD}(n=3)$. (B) Proposed catabolic pathway for the colonic transformation of mono-galloyl-hexoside in humans. Framed names indicated major catabolites.

The compound (-)-epicatechin was initially detected after $4 \mathrm{~h}$ and $8 \mathrm{~h}$ of incubation, respectively ( 8.2 and $0.6 \mu \mathrm{mol} / \mathrm{g} \mathrm{DW})$. The appearance of this compound was marked by a gradual decrease in epigallocatechin, which disappeared completely after $24 \mathrm{~h}$ of incubation (Table 4). Epicatechin can be further transformed into 5-(hydroxyphenyl)- $\gamma$-valerolactones and phenylhydroxyvaleric acid derivatives by dihydroxylation and ring fission transformations [41], but none of these catabolites were detected in our study.

Small quantities of other polyphenols such as syringic acid glucoside 1 and 2, vanillic acid, sinapic acid-glucoside and caffeoyl-hexoside 1 and 2 found in digested mango samples before fermentation disappeared during the faecal incubation. In contrast, low levels of 3-(4-hydroxyphenyl)propanoic acid $(2 \mu \mathrm{mol} / \mathrm{g} \mathrm{DW})$ and 3-phenylacetic acid $(26 \mu \mathrm{mol} / \mathrm{g} \mathrm{DW})$ were detected after 4 and $24 \mathrm{~h}$ respectively of incubation of the digested mango samples with faecal material (Table 4). These levels, increased thereafter, reaching maximum amounts after $48 \mathrm{~h}$ of incubation (19 and $41 \mu \mathrm{mol} / \mathrm{g} \mathrm{DW}$, respectively) (Figure 2A). 


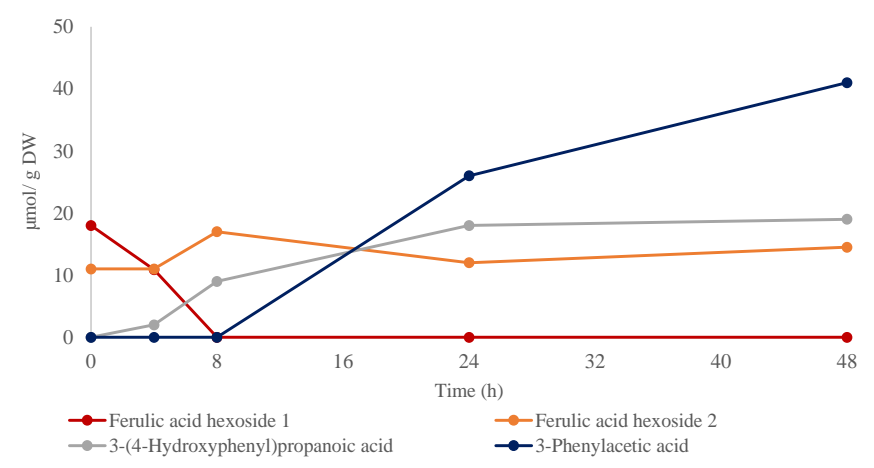

(A)

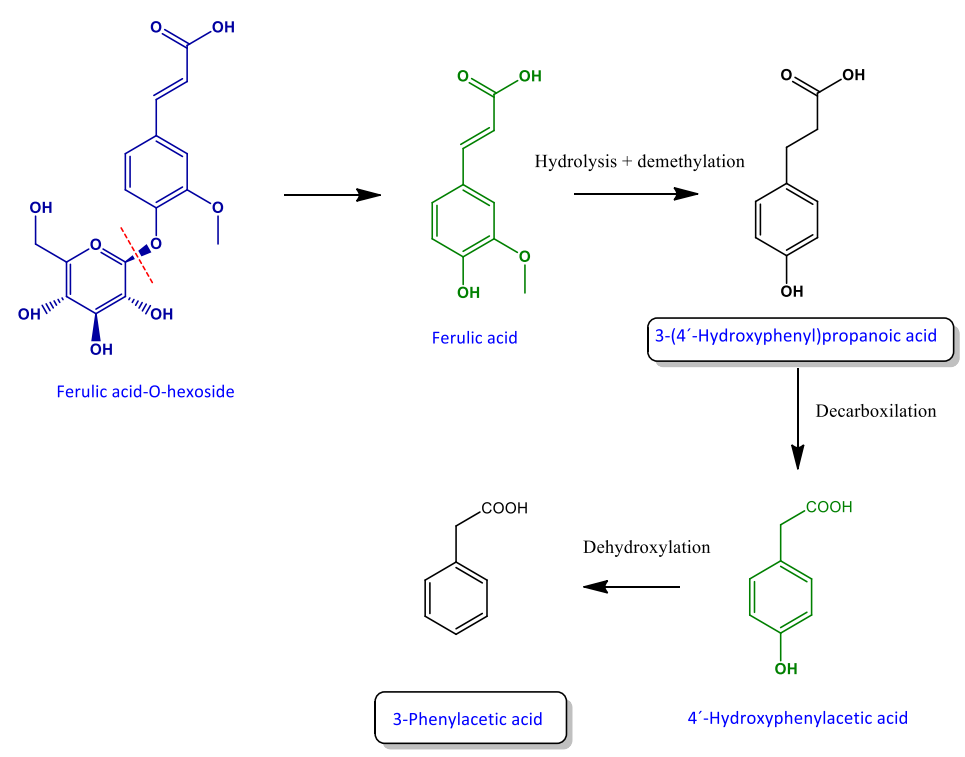

(B)

Figure 2. (A) Ferulic acid-hexoside degradation profiles over $48 \mathrm{~h}$ of incubation with human faeces. Data expressed as $\mu \mathrm{mol} \pm \mathrm{SD}(n=3)$. (B) Proposed catabolic pathway for the colonic transformation of ferulic acid-hexoside in humans. Black: detected metabolites; green; non-detected metabolites. Framed names indicate major catabolites.

These catabolites were arguably formed by the degradation of ferulic acid-hexoside via hydrolysis and the demethylation of ferulic acid to 3-(4-hydroxyphenyl)propanoic acid, and further decarboxylation and dihydroxylation to 3-phenylacetic acid following the procedure described in Figure 2B. Indeed, these catabolites have been reported to be faecal fermentation products of ferulic acid [25].

The incubation of faecal slurries with the digested mango polyphenols also resulted in the appearance of norathyriol after $4 \mathrm{~h}$ of incubation $(0.07 \mu \mathrm{mol} / \mathrm{g} \mathrm{DW})$, the levels decreasing slightly during the fermentation process until disappearing after $48 \mathrm{~h}$ of incubation (Table 4, Figure 3A). This compound has been described as the main microbial-degradation product of mangiferin, in which faecal bacteria act by cleaving the C-glycosyl bond leading to aglycone [42,43] via the pathway illustrated in Figure 3B. The Bacteroides species named MANG, found in human faeces, is reported to be responsible for this transformation [44]. In view of these results, the catabolite norathyriol can be proposed as a biomarker of the colonic degradation of mangiferin.

Overall, the information from this in vitro study is of value because pointed out the great transformation of mango polyphenols through the gastrointestinal tract as well as by the colonic microbiota. The derived catabolites identified in our study merits further investigation to elucidate 
the potential protective effects of mango consumption in vivo. Therefore, these phenolic catabolites will be the focus to the design of ex vivo cell-based experiments (next step) aimed at elucidating the underlying modes of action of the protective effect of mango polyphenol on health.

Moreover, future research will involve the evaluation of the bioavailability of mango pulp polyphenols in humans. The information from the in vivo human studies together with those from this in vitro study would provide a detailed evaluation of the fate of mango pulp polyphenols through the body. Furthermore, this will help to identify additionally potential mango bioactive compounds.

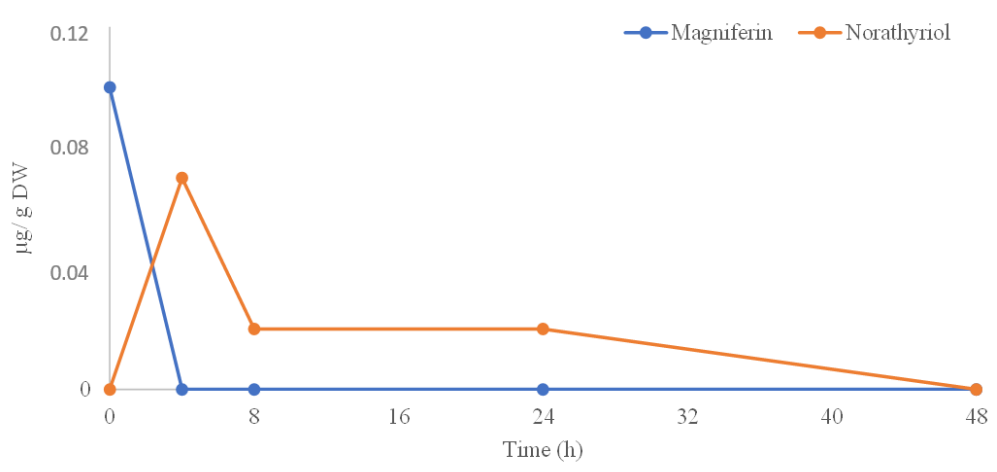

(A)

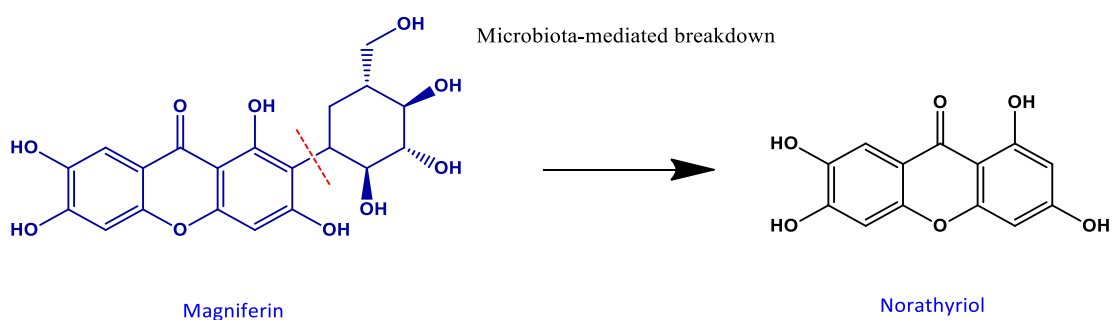

(B)

Figure 3. (A) Mangiferin degradation profiles over $48 \mathrm{~h}$ of incubation with human faeces. Data expressed as $\mu \mathrm{mol} \pm \mathrm{SD}(n=3)$. (B) Proposed catabolic pathway for the colonic transformation of mangiferin in humans.

\section{Conclusions}

The present study investigated the effects of in vitro simulated gastrointestinal digestion and colonic fermentation on the stability and bioaccessibility of mango pulp polyphenols, and evaluated the resultant breakdown products after faecal fermentation by the resident human microbiota. During in vitro gastrointestinal digestion of mango pulp polyphenols there was a significant increase in total polyphenols, with the phenols gallic acid, 3-O-methylgallic acid, methyl gallate, 3,4-dihydroxybenzoic acid and benzoic acid being the most accessible. These are arguably the result of the decrease in other compounds such as monogallyl glucoside and hydroxybenzoic acid hexoside. At colonic level, they were subjected to the action of the microbiota, being converted principally to gallic acid, pyrogallol and 3,4-dihydroxybenoic acid, compounds potentially involved in the health benefits of mango pulp consumption.

Author Contributions: The authors' responsibilities were as follows: Conceptualization: J.M.M.-R. and G.P.-C.; Methodology, Software, Validation and Form Analysis: F.J.R.-G., A.M.-O., J.L.O.-D. and G.P.-C.; Writing-Original Draft Preparation: G.P.-C.; Writing-Review \& Editing: G.P.-C., J.M.M.-R. and V.O.-S.; Visualization: V.O.-S.; Supervision: G.P.-C. and J.M.M.-R.; Project Administration: G.P.-C., V.O.-S., J.M.M.-R.; Funding Acquisition: G.P.-C., V.O.-S., J.M.M.-R. All authors have read and agreed to the published version of the manuscript.

Funding: This study was funded by the Andalusian Institute of Agricultural and Fisheries Research and Training (IFAPA) and the European Rural Development Fund (ERDF, EU) through the Project "Caracterización, biodisponibilidad y potencial saludable de compuestos bioactivos de alimentos" PP.AVA.AVA2019.037 and by the 
European Regional Development Funds (ERDF), the Spanish Ministry of Science, Innovation and Universities (MCIU) and the Spanish State Research Agency (AEI) through the project "Bioavailability of mango polyphenols in healthy and ileostomy patients: relevance of the bioactive forms in vivo on the gastrointestinal health" (RTI2018-096703-J-I00). A. Moreno-Ortega is supported by a predoctoral fellowship funded by the Spanish Ministry of Education, Culture and Sport (FPU16-05881). G. Pereira-Caro was supported by a research contract funded by the project RTI2018-096703-J-I00. J.L. Ordoñez was supported by a research contract funded by the project PP.AVA.AVA2019.037.

Conflicts of Interest: The authors declare no conflict of interest.

\begin{tabular}{|c|c|}
\hline \multicolumn{2}{|c|}{ Abbreviations } \\
\hline UHPLC- & ultra-high-performance liquid chromatography \\
\hline HRMS & coupled to high resolution mass spectrometry \\
\hline FA & formic acid \\
\hline FW & fresh Weight \\
\hline BOD & Before Oral Digestion \\
\hline $\mathrm{AOD}$ & After Oral Digestion \\
\hline AGD & After Gastric Digestion \\
\hline AID & After Intestinal Digestion \\
\hline RT & retention time \\
\hline$[\mathrm{M}-\mathrm{H}]^{-} \operatorname{Exp}$ & experimental exact mass \\
\hline$\Delta$ & mass error \\
\hline OFN & oxygen free nitrogen \\
\hline BMIs & body mass index \\
\hline n.d. & not detected \\
\hline
\end{tabular}

\section{References}

1. Arauz, L. Mango anthracnose: Economic impact and current opinions for integrated management. Plant Dis. 2000, 84, 600-611. [CrossRef]

2. FAOSTAT, 2018. Available online: http://www.fao.org/faostat/es/\#data (accessed on 10 November 2020).

3. Sivakumar, D.; Jiang, Y.; Yahia, E.M. Maintaining mango (Mangifera indica L.) fruit quality during the export chain. Food Res. Int. 2011, 44, 1254-1263. [CrossRef]

4. Kim, Y.; Brecht, J.K.; Talcott, S.T. Antioxidant phytochemical and fruit quality changes in mango (Mangifera indica L.) following hot water immersion and controlled atmosphere storage. Food Chem. 2007, 105, 1327-1334. [CrossRef]

5. Schieber, A.; Ullrich, W.; Carle, R. Characterization of polyphenols in mango puree concentrate by HPLC with diode array and mass spectrometric detection. Innov. Food Sci. Emerg. Technol. 2000, 1, 161-166. [CrossRef]

6. Shabani, Z.; Sayadi, A. The antimicrobial in vitro effects of different concentrations of some plant extracts including tamarisk, march, acetone and mango kernel. J. Appl. Pharma. Sci. 2014, 4, 75.

7. Andreu, G.P.; Delgado, R.; Velho, J.A.; Curti, C.; Vercesi, A.E. Iron complexing activity of mangiferin, a naturally occurring glucosylxanthone, inhibits mitochondrial lipid peroxidation induced by $\mathrm{Fe}^{2+}$-citrate. Eur. J. Pharmacol. 2005, 513, 47-55. [CrossRef] [PubMed]

8. Ramesh, P.R.; Parasuraman, S.; Vijaya, C.; Darwhekar, G.; Devika, G.S. Antidiabetic effect of kernel sedes extract of Mangifera indica (anacardiaceae). Int. J. Pharma Bio Sci. 2001, 2, 385-393.

9. El-Seedi, H.R.; Salem, M.A.; Khattab, O.M.; El-Wahed, A.A.; El-Kersh, D.M.; Khalifa, S.A.; Halabi, M.F. Dietary Xanthones. In Handbook of Dietary Phytochemicals; Springer Nature: Singapore, 2020; pp. 1-22.

10. Duthie, G.G.; Duthie, S.J.; Kyle, J.A.M. Plant polyphenols in cancer and heart disease: Implication as nutritional antioxidants. Nutr. Res. Rev. 2000, 13, 79-106. [CrossRef] [PubMed]

11. Abdullah, A.S.H.; Mohammed, A.S.; Abdullah, R.; Mirghani, M.E.S.; Qubaisi, M.A. Cytotoxic effects of Mangifera indica L. Kernel extract on human breast cancer (MCF-7 and MDA-MB-231 cell lines) and bioactive constituents in the crude extract. BMC Complementary Altern. Med. 2014, 14, 1-10. [CrossRef] [PubMed]

12. Gold-Smith, F.; Fernandez, A.; Bishop, K. Mangiferin and cancer: Mechanisms of action. Nutrients 2016, 8, 396. [CrossRef] [PubMed] 
13. Prabhu, S.; Jainu, M.; Sabitha, K.E.; Devi, C.S. Role of mangiferin on biochemical alterations and antioxidant status in isoproterenol-induced myocardial infarction in rats. J. Ethnopharmacol. 2006, 107, 126-133. [CrossRef] [PubMed]

14. Williamson, G.; Kay, C.D.; Crozier, A. The bioavailability, transport and bioactivity of dietary flavonoids: A review from a historical perspective. Compr. Rev. Food Sci. Food Saf. 2018, 17, 1054-1112. [CrossRef]

15. Burton-Freeman, B.M.; Sandhu, A.K.; Edirisinghe, I. Mangos and their bioactive components: Adding variety to the fruit plate of health. Food Funct. 2017, 8, 3010-3032. [CrossRef]

16. Fang, C.; Kim, H.; Barnes, R.; Talcott, S.T.; Mertens-Talcott, S.U. Daily mango (Mangifera indica L.) consumption for 42 days differentially modulates metabolism and inflammation in lean and obese individuals. FASEB J. 2017, 31, 431.

17. O’Hara, C.; Ojo, B.; Emerson, S.R.; Simenson, A.J.; Peterson, S.; Perkins-Veazie, P.; Payton, M.E.; Hermann, J.; Smith, B.J.; Lucas, E.A. Acute Freeze-Dried Mango Consumption With a High-Fat Meal has Minimal Effects on Postprandial Metabolism, Inflammation and Antioxidant Enzymes. Nutr. Metab. Insights 2019, 12, 1-10. [CrossRef]

18. Blancas-Benitez, F.J.; Mercado-Mercado, G.; Quirós-Sauceda, A.E.; Montalvo-González, E.; González-Aguilar, G.A.; Sáyado-Ayerdi, S.G. Bioaccesibility of polyphenols associated with dietary fiber and in vitro kinetics release of polylphenols in Mexican "Ataulfo" mango (Mangifera indica L.) by-products. Food Funct. 2015, 6, 859-868. [CrossRef]

19. Quirós-Sauceda, A.E.; Sañudo-Barajas, J.A.; Vélez-de la Rocha, R.; Domínguez-Avila, J.A.; Ayala-Zavala, J.F.; Villegas-Ochoa, M.A.; González-Aguilar, G.A. Effects of ripening on the in vitro antioxidant capacity and bioaccessibility of mango cv.'Ataulfo'phenolics. J. Food Sci. Technol. 2019, 56, 2073-2082. [CrossRef]

20. Hernández-Maldonado, L.M.; Blancas-Benítez, F.J.; Zamora-Gasga, V.M.; Cárdenas-Castro, A.P.; Tovar, J.; Sáyago-Ayerdi, S.G. In Vitro Gastrointestinal Digestion and Colonic Fermentation of High Dietary Fiber and Antioxidant-Rich Mango (Mangifera indica L.) "Ataulfo"-Based Fruit Bars. Nutrients 2019, 11, 1564. [CrossRef]

21. Herrera-Cazares, L.A.; Hernández-Navarro, F.; Ramírez-Jiménez, A.K.; Campos-Vega, R.; Reyes-Vega, M.L.; Loarca-Piña, G.; Morales-Sánchez, E.; Wall-Medrano, A.; Gaytán-Martínez, M. Assessment of a functional confectionery added with mango bagasse as vehicle for enhancing bioaccessibility and permeability of phenolic compounds. Food Funct. 2020, 8, 3906-3916. [CrossRef]

22. Kay, C.D.; Clifford, M.N.; Mena, P.; McDougall, G.J.; Andres-Lacueva, C.; Cassidy, A.; Del Rio, D.; Kuhnert, N.; Manach, C.; Pereira-Caro, G.; et al. Recommendations for standardizing nomenclature for dietary (poly) phenol catabolites. Am. J. Clin. Nutr. 2020, 112, 1051-1068. [CrossRef]

23. Moreno-Ortega, A.; Pereira-Caro, G.; Ordóñez, J.L.; Moreno-Rojas, R.; Ortíz-Somovilla, V.; Moreno-Rojas, J.M. Bioaccessibility of Bioactive Compounds of 'Fresh Garlic'and ‘Black Garlic'through In Vitro Gastrointestinal Digestion. Foods 2020, 9, 1582. [CrossRef] [PubMed]

24. De Santiago, E.; Gill, C.I.; Carafa, I.; Tuohy, K.M.; De Peña, M.P.; Cid, C. Digestion and colonic fermentation of raw and cooked Opuntia ficus-indica cladodes impacts bioaccessibility and bioactivity. J. Agric. Food Chem. 2019, 67, 2490-2499. [CrossRef] [PubMed]

25. Pereira-Caro, G.; Borges, G.; Ky, I.; Ribas, A.; Calani, L.; Del Rio, D.; Crozier, A. In vitro colonic catabolism of orange juice (poly) phenols. Mol. Nutr. Food Res. 2015, 59, 465-475. [CrossRef]

26. Alañón, M.E.; Oliver-Simancas, R.; Gómez-Caravaca, A.M.; Arráez-Román, D.; Segura-Carretero, A. Evolution of bioactive compounds of three mango cultivars (Mangifera indica L.) at different maturation stages analyzed by HPLC-DAD-q-TOF-MS. Food Res. Int. 2019, 125, 108526. [CrossRef]

27. Sumner, L.W.; Amberg, A.; Barrett, D.; Beale, M.H.; Beger, R.; Daykin, C.A.; Fan, T.W.; Fiehn, O.; Goldagre, R.; Griffin, J.L.; et al. Proposed minimum reporting standards for chemicals analysis chemical analysis Chemical Analylsis Working Group (CAWG) Metabolomics Standards Initiative (MSI). Metabolomics 2007, 3, 211-221. [CrossRef]

28. López-Cobo, A.; Verardo, V.; Diaz-de-Cerio, E.; Segura-Carretero, A.; Fernández-Gutiérrez, A.; Gómez-Caravaca, A.M. Use of HPLC-and GC-QTOF to determine hydrophilic and lipophilic phenols in mango fruit (Mangifera indica L.) and its by-products. Food Res. Int. 2017, 100, 423-434. [CrossRef]

29. Vithana, M.D.K.; Singh, Z.; Johnson, S.K. Cold storage temperatures and durations affect the concentrations of lupeol, mangiferin, phenolic acids and other health-promoting compounds in the pulp and peel of ripe mango fruit. Postharvest Biol. Technol. 2018, 139, 91-98. [CrossRef] 
30. Oliveira, B.G.; Costa, H.B.; Ventura, J.A.; Kondratyuk, T.P.; Barroso, M.E.; Correia, R.M.; Pimentel, E.F.; Pinto, F.E.; Endringer, D.C.; Romão, W. Chemical profile of mango (Mangifera indica L.) using electrospray ionisation mass spectrometry (ESI-MS). Food Chem. 2016, 204, 37-45. [CrossRef]

31. Meneses, M.A.; Caputo, G.; Scognamiglio, M.; Reverchon, E.; Adami, R. Antioxidant phenolic compounds recovery from Mangifera indica L. by-products by supercritical antisolvent extraction. J. Food Eng. 2015, 163, 45-53. [CrossRef]

32. Palafox-Carlos, H.; Yahia, E.M.; González-Aguilar, G.A. Identification and quantification of majornphenolic compounds from mango (Mangifera indica, cv. Ataulfo) fruit by HPLC-DADMS/MS-ESI and their individual contribution to the antioxidant activity during ripening. Food Chem. 2012, 135, 105-111. [CrossRef]

33. Robles-Sánchez, R.M.; Islas-Osuna, M.A.; Astiazarán-García, H.; Vázquez-Ortiz, F.A.; Martín-Belloso, O.; Gorinstein, S. Quality index, consumer acceptability, bioactive compounds, and antioxidant activity of fresh cut "Ataulfo" mangoes (Mangifera indica L.) as affected by low temperature storage. J. Food Sci. 2009, 74, S126-S134. [CrossRef]

34. Robles-Sánchez, R.M.; Rojas-Graü, M.A.; Odriozola-Serrano, I.; González-Aguilar, G.A.; Martín-Belloso, O. Effect of minimal processing on bioactive compounds and antioxidant activity of fresh-cut "Kent" mango (Mangifera indica L.). Postharvest Biol. Technol. 2008, 51, 384-390.

35. Abbasi, A.M.; Guo, X.; Fu, X.; Zhou, L.; Chen, Y.; Zhu, Y.; Yan, H.; Liu, R.H. Comparative assessment of phenolic content and in vitro antioxidant capacity in the pulp and peel of mango cultivars. Int. J. Mol. Sci. 2015, 16, 13507-13527. [CrossRef]

36. Rodríguez-Roque, M.J.; Rojas-Graü, M.A.; Elez-Martínez, P.; Martín-Belloso, O. Soymilk phenolic compounds, isoflavones and antioxidant activity as affected by in vitro gastrointestinal digestion. Food Chem. 2013, 136, 206-212. [CrossRef]

37. Lucas-González, R.; Viuda-Martos, M.; Álvarez, J.A.P.; Fernández-López, J. Changes in bioaccessibility, polyphenol profile and antioxidant potential of flours obtained from persimmon fruit (Diospyros kaki) co-products during in vitro gastrointestinal digestion. Food Chem. 2018, 256, 252-258. [CrossRef]

38. Bohn, T. Dietary factors affecting polyphenol bioavailability. Nutr. Rev. 2014, 72, 429-452. [CrossRef]

39. Quirós-Sauceda, A.E.; Chen, C.Y.O.; Blumberg, J.B.; Astiazaran-Garcia, H.; Wall-Medrano, A.; González-Aguilar, G.A. Processing 'ataulfo'mango into juice preserves the bioavailability and antioxidant capacity of its phenolic compounds. Nutrients 2017, 9, 1082. [CrossRef]

40. Sandhu, A.; Fan, J.; Xiao, D.; Edirisinghe, I.; Burton-Freeman, B. Identification of phenolic metabolites in human plasma and urine after mango consumption. Curr. Dev. Nutr. 2019, 3 (Suppl. 1), 021-19. [CrossRef]

41. Chen, W.; Zhu, X.; Lu, Q.; Zhang, L.; Wang, X.; Liu, R. C-ring cleavage metabolites of catechin and epicatechin enhanced antioxidant activities through intestinal microbiota. Food Res. Int. 2020, 135, 109270. [CrossRef]

42. Li, Y.; Meselhy, M.R.; Wang, L.Q.; Nakamura, N.; Hattori, M. Biotransformation of a C-Glycosylflavone, Abrusin 2"-O- $\beta$-D-Apioside, by Human Intestinal Bacteria. Chem. Pharm. Bull. 2000, 48, 1239-1241. [CrossRef]

43. Souza, J.R.; Trevisan, M.T.S.; Feitosa, J.P.; Ricardo, N.M.; Hull, W.E.; Erben, G.; Wurtele, G.; Breuer, A.; Frei, E.; Ulrich, C.M.; et al. Transformation of Mangiferin to Norathyriol by Human Fecal Matrix in Anaerobic Conditions: Comprehensive NMR of the Xanthone Metabolites, Antioxidant Capacity, and Comparative Cytotoxicity Against Cancer Cell Lines. Nat. Prod. Commun. 2020, 15, 1-9.

44. Sanugul, K.; Akao, T.; Li, Y.; Kakiuchi, N.; Nakamura, N.; Hattori, M. Isolation of a human intestinal bacterium that transforms mangiferin to norathyriol and inducibility of the enzyme that cleaves a C-glucosyl bond. Biol. Pharm. Bull. 2005, 28, 1672-1678. [CrossRef]

Publisher's Note: MDPI stays neutral with regard to jurisdictional claims in published maps and institutional affiliations.

(C) 2020 by the authors. Licensee MDPI, Basel, Switzerland. This article is an open access article distributed under the terms and conditions of the Creative Commons Attribution (CC BY) license (http://creativecommons.org/licenses/by/4.0/). 